\title{
The Role of Neuropeptides in Suicidal Behavior: A Systematic Review
}

\author{
Gianluca Serafini, ${ }^{1}$ Maurizio Pompili, ${ }^{1}$ Daniel Lindqvist, ${ }^{2}$ \\ Yogesh Dwivedi, ${ }^{3}$ and Paolo Girardi ${ }^{1}$ \\ ${ }^{1}$ Department of Neurosciences, Mental Health and Sensory Organs, Suicide Prevention Center, Sant'Andrea Hospital, \\ Sapienza University of Rome, 1035-1039 Via di Grottarossa, 00189 Rome, Italy \\ ${ }^{2}$ Department of Clinical Sciences, Section of Psychiatry, Lund University, SE-221 85 Lund, Sweden \\ ${ }^{3}$ Psychiatric Institute, Department of Psychiatry, College of Medicine, University of Illinois at Chicago, Chicago, IL 60612, USA
}

Correspondence should be addressed to Gianluca Serafini; gianluca.serafini@uniromal.it

Received 27 April 2013; Revised 1 July 2013; Accepted 3 July 2013

Academic Editor: Rajnish Chaturvedi

Copyright (C) 2013 Gianluca Serafini et al. This is an open access article distributed under the Creative Commons Attribution License, which permits unrestricted use, distribution, and reproduction in any medium, provided the original work is properly cited.

There is a growing evidence that neuropeptides may be involved in the pathophysiology of suicidal behavior. A critical review of the literature was conducted to investigate the association between neuropeptides and suicidal behavior. Only articles from peer-reviewed journals were selected for the inclusion in the present review. Twenty-six articles were assessed for eligibility but only 22 studies were included. Most studies have documented an association between suicidality and some neuropeptides such as corticotropin-releasing factor (CRF), VGF, cholecystokinin, substance P, and neuropeptide Y (NPY), which have been demonstrated to act as key neuromodulators of emotional processing. Significant differences in neuropeptides levels have been found in those who have attempted or completed suicide compared with healthy controls or those dying from other causes. Despite cross-sectional associations between neuropeptides levels and suicidal behavior, causality may not be inferred. The implications of the mentioned studies were discussed in this review paper.

\section{Introduction}

Suicide represents one of the leading causes of premature death in the general population. Higher suicide rates have generally been reported in men compared to women in almost all countries, with elderly men at especially high risk for completed suicide [1-3]. Rates of attempted suicide average 20-30 times higher than rates of completed suicide in the general population but are probably underreported and range widely in method and seriousness of intent $[4,5]$. Most cases of suicide involve psychiatric conditions, nearly half of which (48.5\%) suffered from mood disorders [6].

Major depressive disorder (MDD) is a chronic and invalidating disease associated with significant functional impairment and occupational disability. Individuals with MDD are at increased risk for suicidal behavior [7-13]. Impulsivity and loss of impulse control may be considered important dimensions of suicidality. As opposed to psychiatric disorders such as MDD that may be regarded as more proximal risk factors for suicide, some have suggested that impulsivity may be seen as a personality characteristic and thus a more distal risk factor for suicide [14]. Yet others have emphasized the importance to distinguish between attempt impulsivity (state) and attempter impulsivity (trait) in understanding suicide attempts [15]. Impulsive personality characteristics have been associated with a higher likelihood of suicidal behavior across nosological entities and also in nonpsychiatric populations [16]. The structure of impulsivity in severe depression as well as its relationships to suicide attempts have been investigated by Corruble et al. [17]. They found that recent suicide attempts in severely depressed individuals were related to loss of control and cogntive impulsivity but not to nonplanning. The tendency to engage in more impulsive behaviors, reflecting possible loss of impulse control, may be assessed using the impulse control scale [18]. Behavioral loss of control has been found as a state-dependent dimension of impulsivity 
and suicide attempts were mainly related to loss of control in depressed patients. The planning subscale of the suicide intent scale [19] can be used to objectify degree of impulsivity during a suicide attempt.

Despite the recent advancements in the understanding of suicide, our current knowledge concerning the neurobiology of this complex phenomenon is still limited.

Peptide hormones might play a critical role in normal as well as pathological functions of the central nervous system $[20,21]$ acting as selective messengers in specific emotive circuits of the brain [22] and function as crucial mediators of the stress response and adaptation [23]. Neuropeptides show high specificity for their target receptors and are highly potent; also, they are not accumulated in tissues as they are efficiently metabolized by endogenous enzymes [20]. They generally interact with metabotropic or G-protein-coupled receptors, whereas neurotransmitters are chemical substances that affect the excitability of neurons acting as mediators of the neurotransmission through synapses. Neuropeptides are generally stored in large dense-core vesicles; conversely, neurotransmitters are secreted in small synaptic vesicles mainly located in clusters at presynaptic locations. It has been suggested that peptide signals play a prominent role in the information processing, that is, quite unlike that of conventional neurotransmitters [24].

Neuropeptides may modulate a more prolonged transmission than typical neurotransmitters showing relevant neurotrophic effects [25]. Neuropeptides are mainly associated with one or more neurotransmitters which serve to integrate their modulatory functions. The high frequency release of neuropeptides is in line with the ability to mediate adaptation to homeostatic challenges [23]. Some authors suggested that cholecystokinin (CCK) and neuropeptide Y (NPY) are promising options for the discovery of intriguing and more selective pharmacological options [26]. Other neuropeptides like corticotropin-releasing factor (CRF) and neurokinin 1 (NK1) substance P systems are known to modulate 5HT neurons through actions within the dorsal raphe (DR) networks [27]. A hyperactivity of central neuropeptidergic circuits such as CRF and arginine vasopressin (AVP) systems may play a key role in major affective and anxiety disorders [25, 28, 29]. These neuropeptidergic systems may be involved in the action of antidepressant drugs, as demonstrated by the paroxetineinduced reduction of vasopressinergic overexpression [30]. Neuropeptides dysregulation may be also involved in suicidal behavior. Sherrin et al. [31] compared CCKB receptor gene expression in ten suicide victims compared with ten matched controls using quantitative PCR and showed increased gene expression in suicide victims in prefrontal and cingulate cortex as well as cerebellum. This study was in line with previous results demonstrating increased CCK receptor binding in frontal and cingulated cortex of suicide victims [32]. Also, recent suicide attempts have been associated with lower plasma levels of NPY [33]. Lower NPY levels have been found in prefrontal cortex and caudate nucleus of suicide victims compared to age-matched subjects with other causes of death [34]. Reduced NPY levels, which are widely distributed throughout the central nervous system, have been found in depressed patients compared to controls [35], as well as in the brain of suicide victims compared to those who died a natural or accidental death [34].

To what extent neuropeptide abnormalities may be considered reliable predictors of suicidal behavior is, however, a matter of debate. Considering this background, we aimed to critically review the current literature about the role of neuropeptides in suicidal behavior.

\section{Methods}

2.1. Information Sources, Search Strategy, and Study Selection. A detailed search strategy summarized in Figure 1 was used to identify relevant studies. The possible involvement of neuropeptides in suicidal behavior was investigated using a detailed PubMed/MEDLINE, Scopus, PsycLit, and PsycInfo search to identify all papers and book chapters in English language during the period between January 1985 and January 2013. The search used a combination of the following terms: "Neuropeptides" OR “Central nervous System Peptides” OR "Small bioactive peptides" AND "Suicid*" (including suicidal behaviors OR suicide ideation OR suicidal thoughts OR deliberate self-harm OR suicidal attempts). Two independent researchers conducted a two-step literature search. Any discrepancies between the two reviewers who, blind to each other, examined the studies for the possible inclusion were resolved by consultations with a senior author. The reference lists of the articles included in the review were also manually checked for relevant studies.

Studies were included according to the following criteria: (a) being an original paper in a peer-reviewed journal and (b) have analyzed the possible involvement of neuropeptides in suicidal behavior. Figure 1 summarized the search strategy used for selecting studies (identification, screening, eligibility, and inclusion process) in the review.

2.2. Study Design and Eligibility Criteria. To achieve a high standard of reporting, we have adopted Preferred Reporting Items for Systematic Reviews and Meta-Analyses, (PRISMA) guidelines [36]. The PRISMA Statement consists of a 27-item checklist and a four-phase flow diagram for reporting in systematic reviews and meta-analyses. PRISMA includes the broader effort to improve the reporting of different types of health research and in turn to improve the quality of research used in decision making in healthcare.

2.3. Recorded Variables. The recorded variables for each article about neuropeptides and suicide were sample characteristics, study design, type of neuropeptide which has been investigated, main findings, limitations, and conclusions (Table 2).

\section{Results}

3.1. Number of Studies Selected. The combined search strategies yielded a total of 185 articles of which, after a complete analysis, 26 full-text articles were screened and considered for the inclusion in the current review. 159 studies were excluded because they were considered not relevant to the main topic. Specifically, we excluded articles not published in peer 


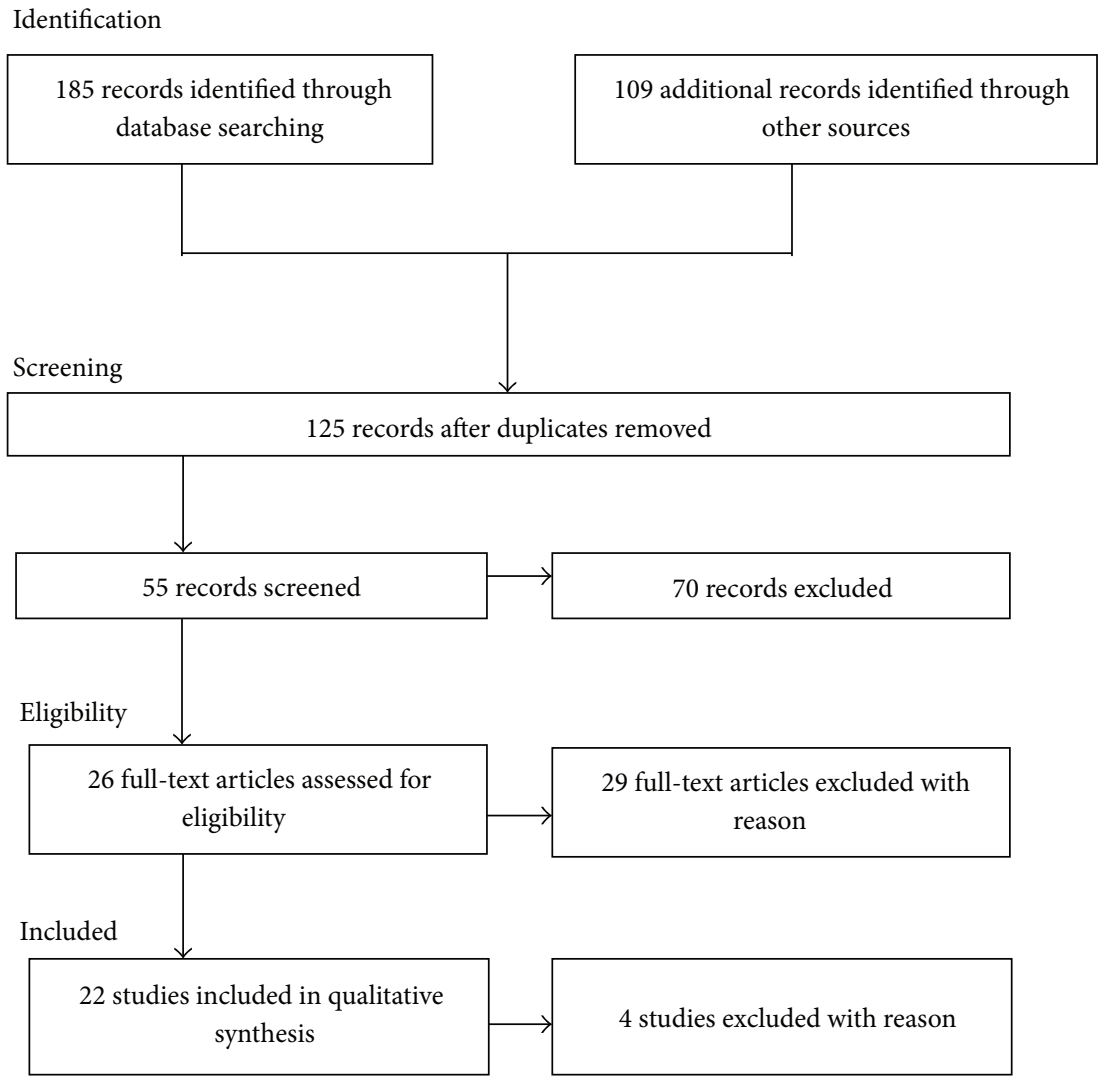

FIGURE 1: Flowchart of the search and selection process identification.

reviewed journals and not in English language, articles without abstracts, abstracts that did not explicitly mention the link between neuropeptides and suicide, articles with a publication date before 1985, and those with unclear data regarding materials and methods and number of patients analyzed. As mentioned, we assessed 26 articles for eligibility but 4 full-text articles were excluded due to low relevance to the main theme leaving 22 studies that fulfilled our inclusion criteria.

3.2. Corticotropin-Releasing Factor (CRF), Oxytocin, and Arginine Vasopressin (AVP). CRF, oxytocin, and AVP have been hypothesized to play a crucial role in the pathogenesis of major affective disorders and presumably suicidal behavior.

Clinical and basic research supports the notion that these neuropeptides appear crucial in modulating behavior. During the past decade, drug discovery efforts using neuropeptide receptor ligands have focused on a limited number of neuropeptides, such as CRF, oxytocin, and AVP although many other neuropeptide systems have been identified. Table 1 summarized the large body of evidence suggesting the involvement of CRF, oxytocin, and AVP in major affective disorders and suicidal behavior.

3.3. Galanin. Galanin (GAL) is a 30-amino-acid peptide that binds GAL receptor1 (R1), GALR2, and GALR3 found along the HPA axis and involved in several human functions such as food and alcohol intake, metabolism, osmoregulation, seizure threshold, and reproduction. Depression-like behaviors seem to be induced by the activation of GALR1 and attenuated depression-like behaviors derived by the activation of GALR3 [103]. GAL immunoreactivity has been showed in human midbrain and limbic areas [104]. Although several studies sustained a role of GAL in the hypothalamic-pituitary-adrenal (HPA) axis activity, findings about this association are quite conflicting [105]. GAL has been reported to modulate serotonergic and noradrenergic circuits [106] as well as GAL transmission (related to the inhibition of dopaminergic neurons in the ventral tegmental area) mediating anhedonia and reduced locomotor activity in depressed patients enhanced locus coeruleus (LC) hyperactivity [107]. GAL antagonists have been reported to improve these effects [107]. The expression of somatodendritic 5HTla autoreceptors (significantly involved in the pathophysiology of major depression) in the DR nucleus may be modified by GAL with a consequent enhancement of serotonergic transmission [108]. Anxiolytic and antidepressant properties have been reported using GALR3 antagonists in animal models [109]. Finally, evidence suggested that activation of GALR2 is associated with neurogenesis and neuroprotective effects improving hippocampal damage which was commonly found after chronic depression [110].

3.4. Substance $P$. SP that binds three different g-proteincoupled tachykinin receptors-NK1, NK2, and NK3, has been 
TABLE 1: Neuropeptides that may be relevant in major affective disorders and suicidal behavior.

CRF is a 41-amino-acid neuropeptide that binds the G-protein-coupled receptors CRF1 and CRF2. The association between CRF/CRF1 system and stress-related disorders is well known [23, 37-39]. CRF is mainly implicated in the regulation of the HPA axis, the abnormalities (increased cerebral spinal fluid CRF, hyperreactivity of the HPA axis, basal hypercortisolemia, increased release of adrenocorticotropin hormone (ACTH) by CRF in subjects treated with dexamethasone, and reduced corticosteroid receptor mediated feedback inhibition of the HPA axis) of which have been frequently associated with psychiatric conditions [40]. It has been demonstrated that antidepressant treatment may restore HPA-axis abnormalities [41], particularly in the paraventricular nucleus of the hypothalamus [42],

Corticotropinreleasing factor

Arginine vasopressin

Oxytocin enhancing the corticosteroid receptor (CR) hypothesis based on the impaired CR function in the pathophysiology of major depression [43]. The hyperactivity and dysregulation of CRF system may trigger and/or involve the maintenance of these alterations as chronic stress or depression have been associated with increased limbic levels of CRF [44-46]. The hyperactivity of CRF system has been associated with reduced hypophyseal CRF binding in depressed patients [47] and reduced corticosteroid feedback inhibition [48]. Moreover, CRF is also implicated in emotional regulation, learning and memory, and autonomic and monoaminergic modulation which are involved in the pathogenesis of major depression [37, 49]. Relevantly, CRF antagonism modulates alterations in many other neuropeptides and hormones systems [30]. Overall, there is a large body of evidence suggesting the involvement of CRF in the pathophysiology of major affective disorders [50-53] but not all studies replicated these findings [54].

AVP is a nine-amino-acid neuropeptide released by the magnocellular terminals into the posterior pituitary mediating the resorption of water in the kidney. AVP interacts with CRF in the parvocellular neurons with the final aim to modulate HPA-axis activity. AVP transmission is also involved in different functions such as learning and memory, aggression, and sociality [55]. AVP binds Vla, V1b, and V3 G protein-coupled receptors which are associated with vasoconstriction, ACTH release, and antidiuresis. Parvocellular AVP and CRF are known to regulate the HPA axis.

Several findings including those derived by postmortem analyses suggested that AVP was increased in either the brain or plasma of depressed patients $[56,57]$. Stressful stimuli lead to persistent increase of AVP in CRF neurons without inducing CRF changes [58]. ACTH release and responsiveness induced by CRF effects are enhanced by AVP [59]. Dinan and Scott [60] suggested that prolonged AVP transmission is a critical mechanism in determining HPA axis hyperactivity in depressed patients. There is evidence that genetic variation of AVP as well as its genetic inactivation in animals $[61,62]$ was related to childhood-onset mood disorders and anxiety-related behaviors. Furthermore, AVP antagonists have demonstrated efficacy in anxious and depressive human conditions showing anxiolytic and antidepressant properties $[63,64]$. Overall, there are consistent evidence suggesting an active involvement of AVP in the pathogenesis of major affective disorders and recently several AVP antagonists showed promising results as possible antidepressant and anxiolytic medications [65].

Oxytocin is a nonapeptide binding to a single G-protein coupled receptor and mainly implicated in behaviors such as childbirth, lactation and sexual behaviors, social memory, and cognition. Oxytocin is synthesized in the paraventricular and supraoptic nuclei of the hypothalamus, transported to the posterior pituitary and released to amygdala, hypothalamus, hippocampus, and nucleus accumbens. According to animal studies, the infusion of oxytocin stimulates maternal behavior and the administration of antagonists inhibits this behavior [66]. Social memory has been demonstrated to be influenced by the oxytocin system in laboratory animals and humans. Oxytocin antagonist may inhibit social recognition in rats that was enhanced by the infusion of an oxytocin agonist into the lateral ventricles [67]. Findings from animal studies suggested that oxytocin represents a critical modulating neuropeptide in the regulation of social interaction. Recently, it has been demonstrated that recognition memory for faces but not nonsocial stimuli has been enhanced in humans by intranasal administration of oxytocin [68]. Furthermore, oxytocin both in rodents [69] and human subjects [70] may influence the stress response modulating the HPA axis and reducing the release of stress hormone. The anxiolytic activity of oxytocin and oxytocin agonists that may be inhibited by oxytocin antagonists has been also reported in several preclinical tests [71, 72]. Intranasal oxytocin has been suggested to reduce the activation of the amygdala in response to fearful or threatening scenes in human individuals [73]. Reduced plasma levels of oxytocin have been found in patients with MDD [74] and in the cerebrospinal fluid (CSF) of schizophrenic patients [75]. Oxytocin agonists have also been reported to be effective in preclinical tests of antidepressant activity such as the tail suspension test [72]. However, CSF oxytocin has also been suggested as an important modulator of suicidal intent and interpersonal violence. Jokinen et al. [76] found that 28 medication-free suicide attempters had lower CSF oxytocin levels compared to 19 healthy controls. Among the suicide attempters, CSF oxytocin levels correlated significantly and negatively with the planning subscale of the Beck Suicide Intent Scale (SIS). After regression analyses, suicide intent was a significant predictor of CSF oxytocin corrected for age and gender whereas lifetime violent behaviour showed a trend to be a predictor of CSF oxytocin. Moreover, oxytocin agonists have been reported to restore prepulse inhibition that is disrupted by $\mathrm{N}$-methyl-D-aspartate (NMDA) antagonists or dopamine agonists in rats [72, 77]. Intranasal administration of oxytocin was also reported to be useful as an adjunctive treatment in patients with schizophrenia who were treated with antipsychotic medications [78]. Finally, reduced oxytocin plasma levels have been demonstrated in autistic children [79] and several single nucleotide polymorphisms in the oxytocin system have been associated with autism [80]. Speech comprehension and social recognition [81] as well as repetitive behaviors [82] may be improved in autistic patients with administration of oxytocin. 


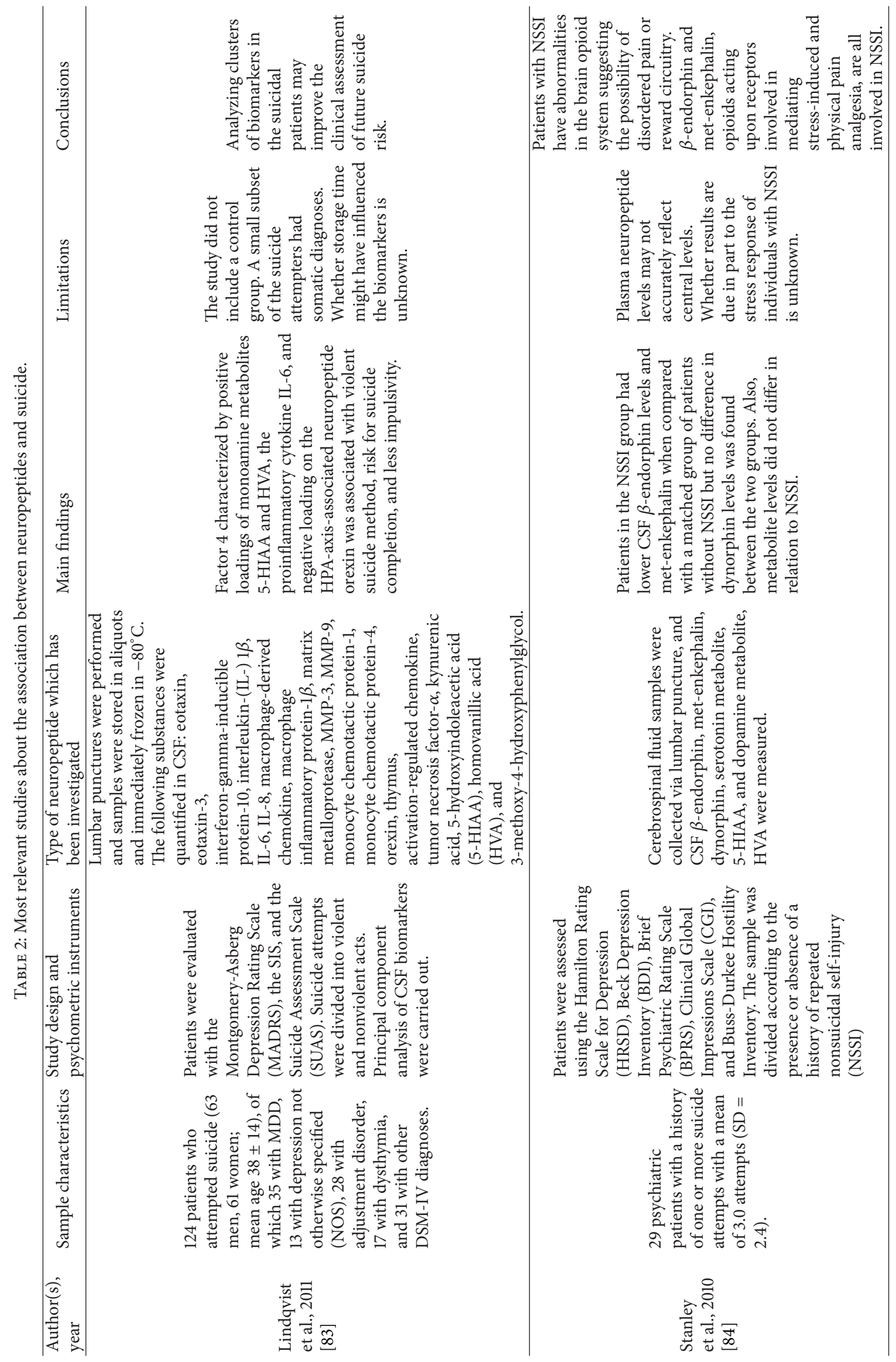




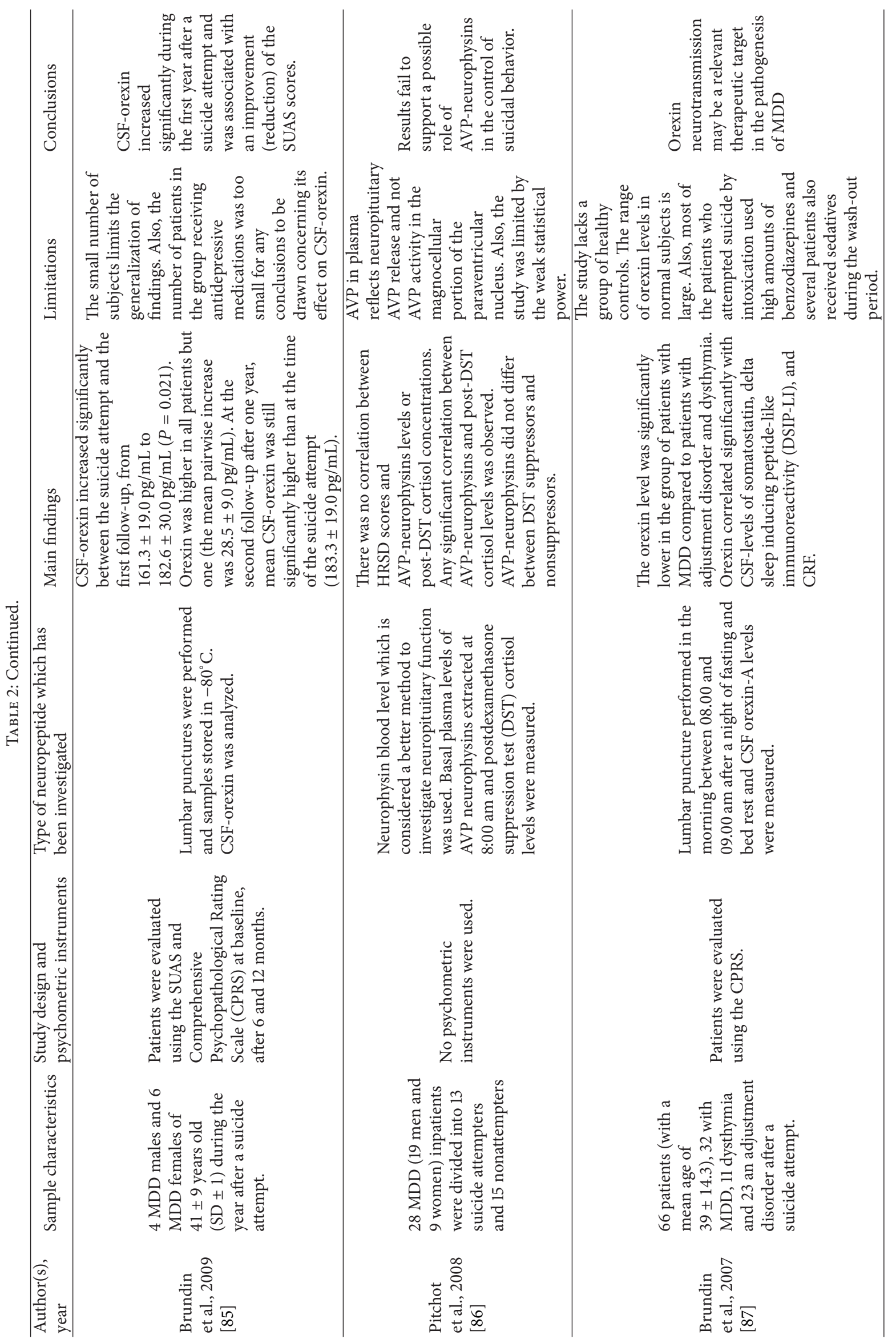




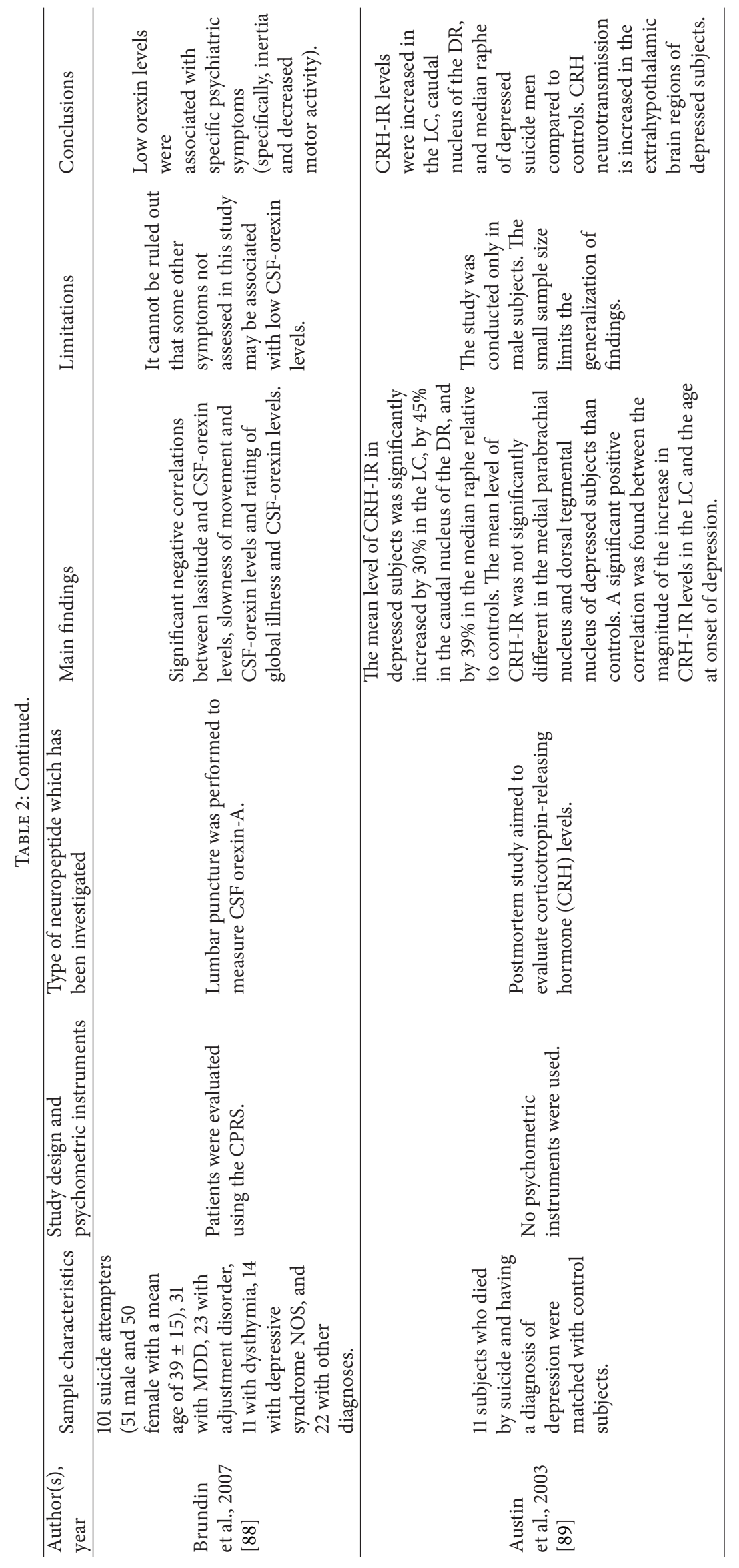




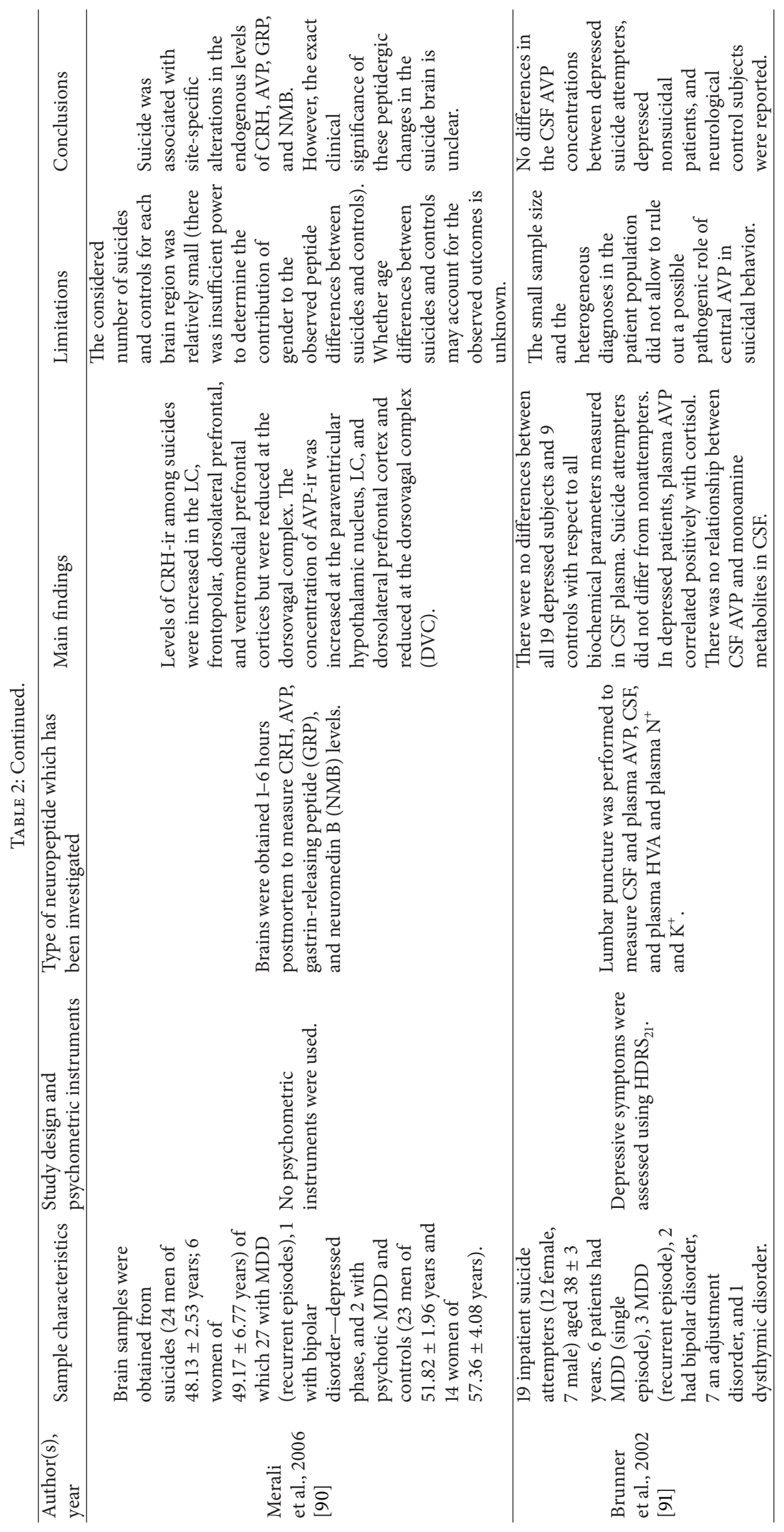




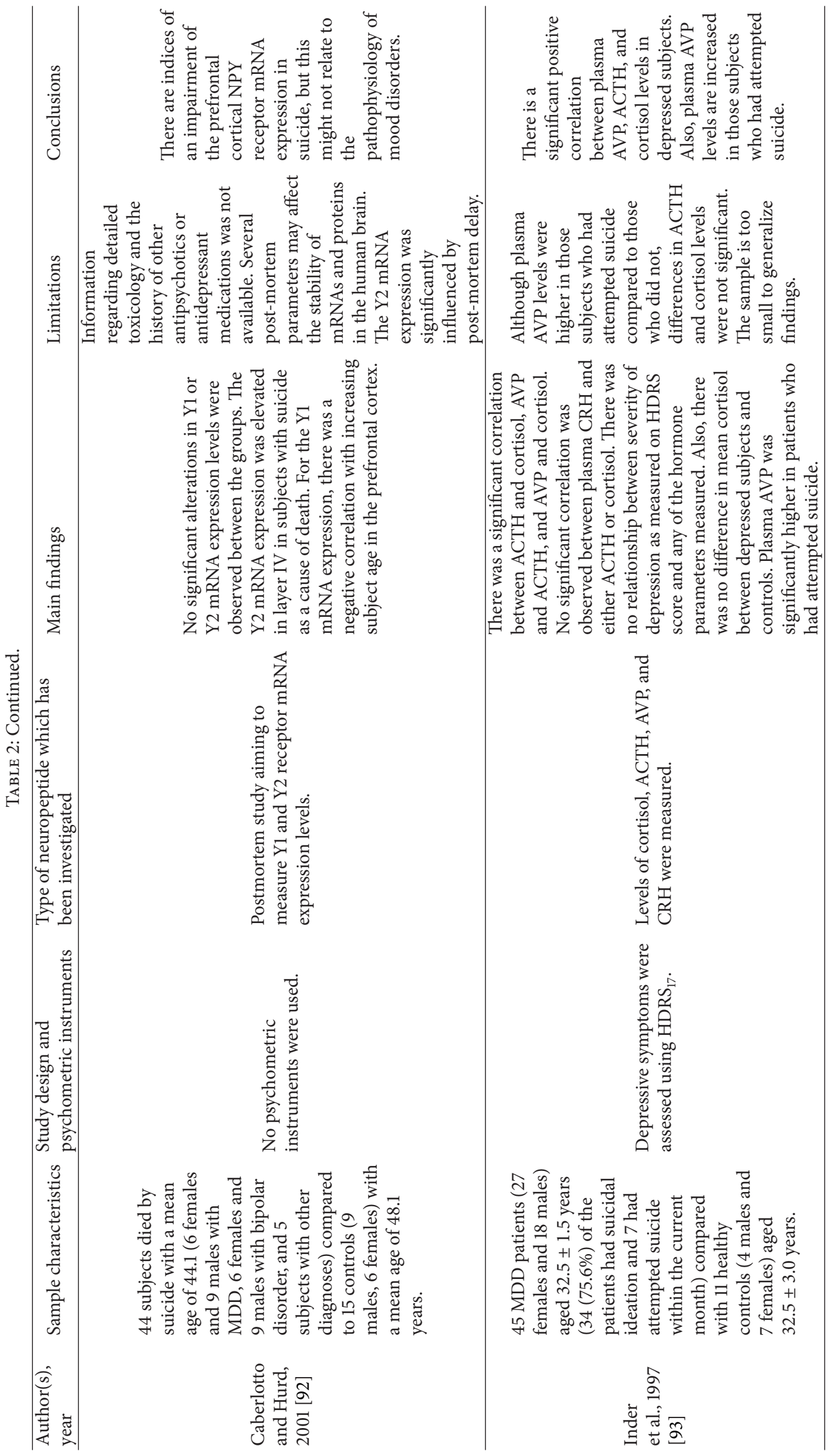




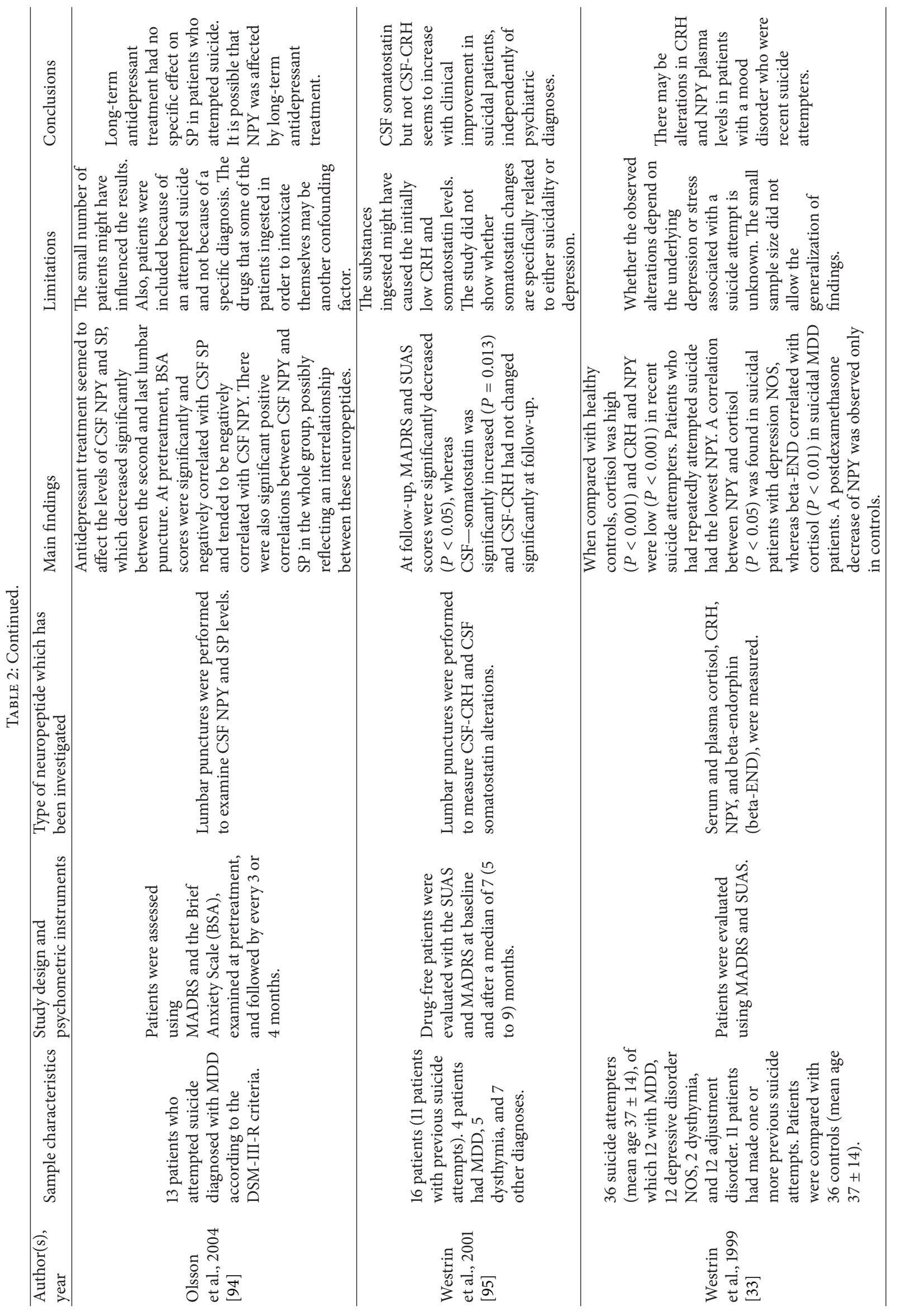




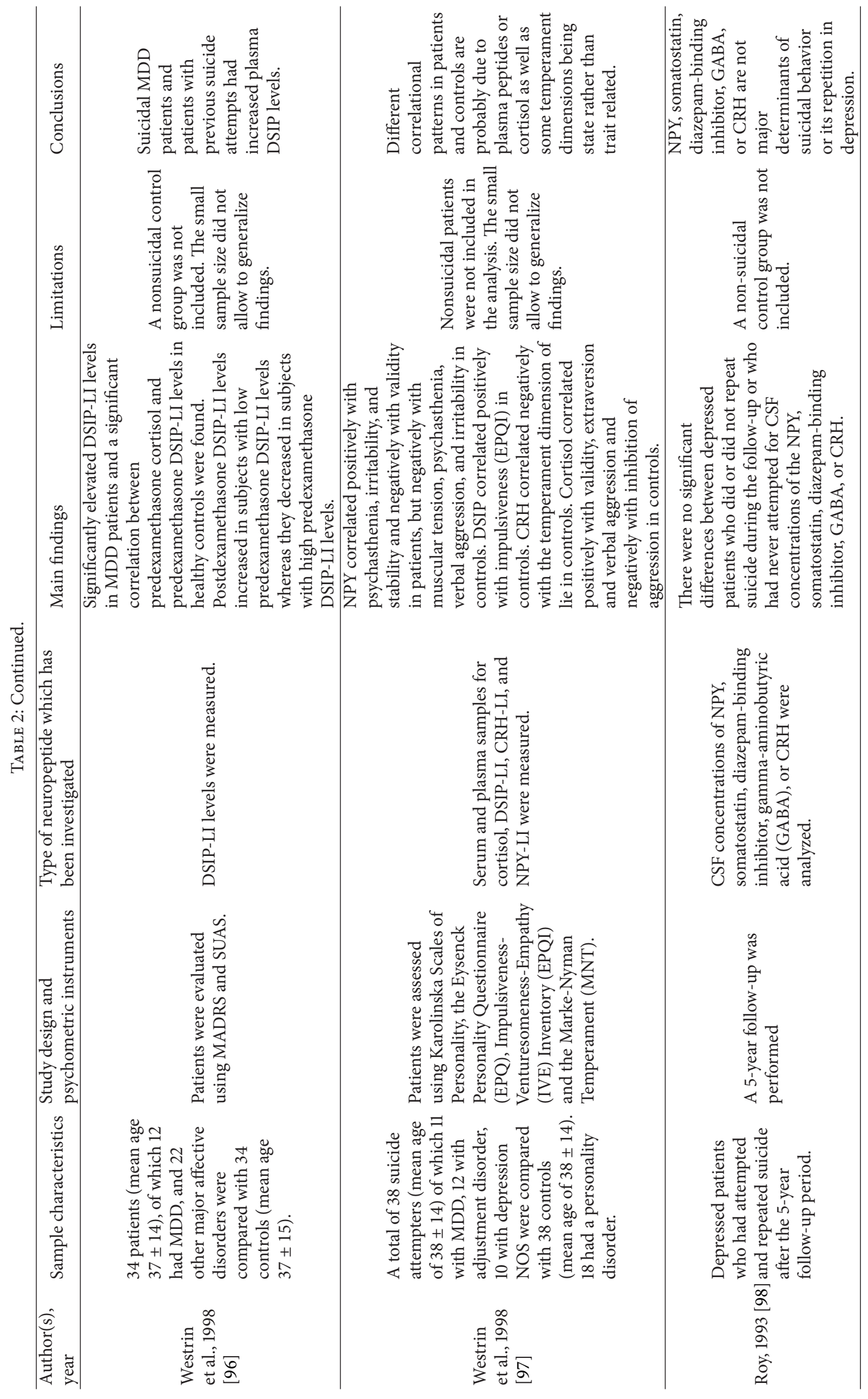




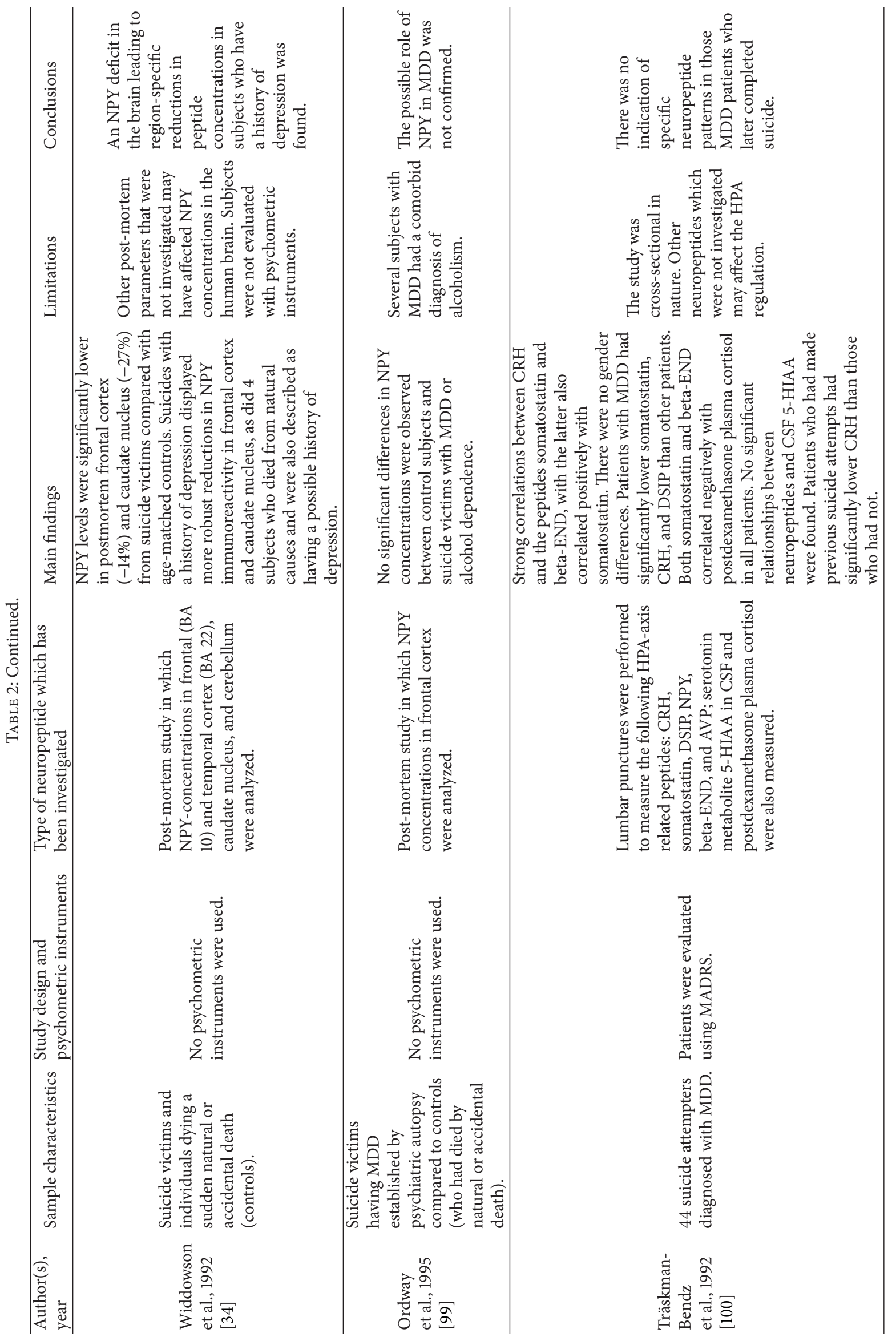




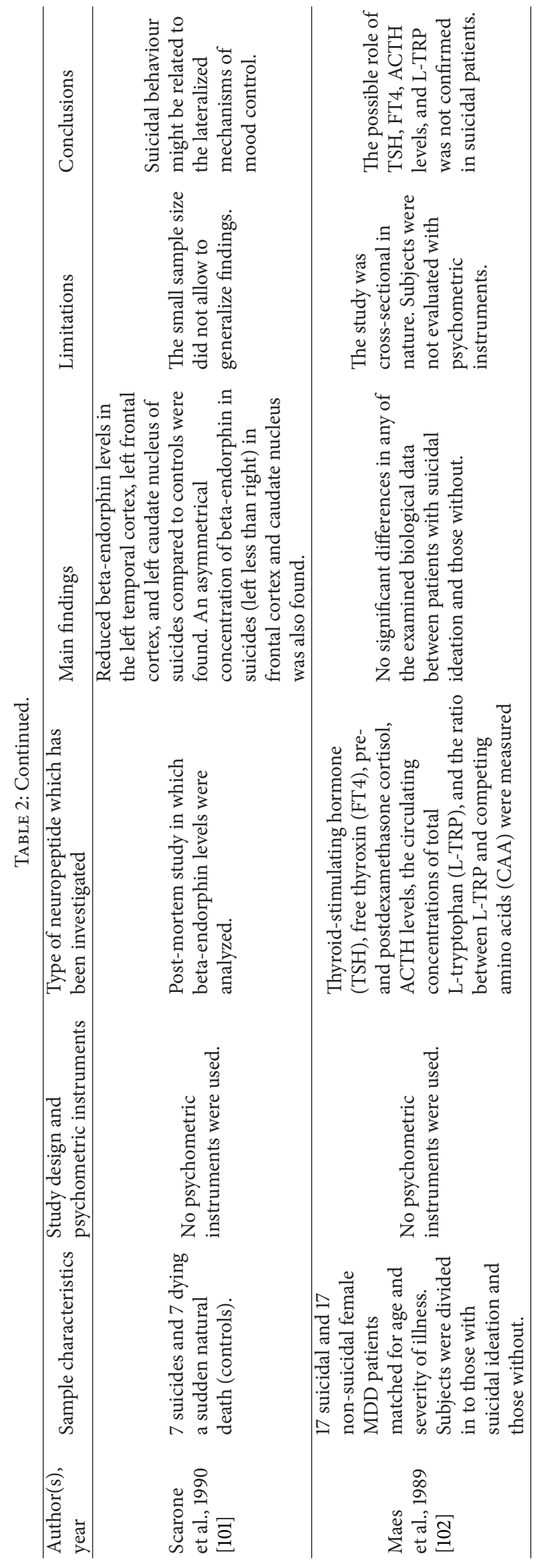


associated with nociception, respiration, cardiovascular and thermoregulation, gut motility, emetic response, and stressrelated disorders [111]. The SP/NK1 system modulates central noradrenergic and serotonergic circuits. Serotonergic activity in the hippocampus and lateral septum was enhanced by NK1 antagonism [112]. NK1 immunoreactivity has been found in the noradrenergic neurons of LC [113]. Several studies sustained a role of SP/NK1 system in the HPA axis and stress responsiveness but not all studies confirmed these findings [111]. Stressful situations may stimulate SP/NK1 system and enhance SP release in the hypothalamus, amygdala, lateral septum, nucleus accumbens, and LC [114]. Animal models have shown that NK1 antagonist may reduce anxiogenic effects related to the injection of SP in the central nucleus of the amygdala [115] showing antidepressant properties [116]. Furmark et al. [117] using functional imaging also suggested that NK1 antagonism in humans reduced social anxiety related to the modulation of serotonergic circuits. NK1 antagonists represent promising medications with consistent antidepressant and anxiolytic properties. There are, however, conflicting results based on the main studies $[114,115]$ measuring SP levels among psychiatric patients and controls. Animal models of anxiety and depression have shown preclinical efficacy by NK2 antagonists [118] whereas NK3 receptors might be valid targets for antipsychotic treatment [119].

3.5. Neuropeptide Y. NPY is a 36-amino-acid which is involved in circadian rhythms, neurogenesis and neuroprotection, nociception, feeding behavior and energy regulation, neuronal excitability, emotion and cognition, stress response, and resilience. NPY action is associated with several neurotransmitters such as GABA, serotonin, norepinephrine, and other catecholamines $[120,121]$. The central NPY transmission has been associated with anxiolytic and antidepressant properties in animal models [122]. Anxiolytic and antidepressant effects have been demonstrated after the activation of receptors Y1 in animals [123] whereas the activation of Y2 is related to anxiogenic effects [124]. Recently, conflicting results have emerged after activation or Y5 antagonism [125].

The enhanced NPY transmission might be stimulated to control stress response through the modulation of CRF and noradrenergic tone. In fact, the reduced NPY transmission may be related to increased CRF and noradrenergic transmission determining anxiety and depression behaviors [126]. NPY, no doubt, has important and complex interactions with the CRF system. Sajdyk et al. [127] suggested that NPY is associated with anxiolytic activity in the amygdala where both CRF receptors and high concentrations of NPY have been found. However, mixed findings regarding NPY levels have been reported among humans [128].

3.6. Cholecystokinin. CCK is a 33-amino-acid peptide that has been found to be implicated in gastric emptying, gallbladder contraction, pancreatic enzyme release, and suppression of appetite. CCK levels were observed in the cortex, hippocampus, amygdala, nucleus accumbens, striatum, and substantia nigra [129]. CCKR1 and CCKR2, with the latter predominantly expressed in the brain, have been described as the $2 \mathrm{G}$-protein coupled receptors binding CCK. Relevant effects on behaviors which are known to be mediated via dopamine seem to be induced by CCK peptides.

The interaction of CCK with CCKR2 has been reported to inhibit dopamine release blocking dopamine-mediated behaviors into the anterior nucleus accumbens whereas the binding with CCKR1 into the posterior nucleus accumbens is associated with opposite effects $[130,131]$. CCK agonists that may be active in the treatment of schizophrenia should be nonselective or CCKR1 selective. Overall, there are evidence suggesting that CCK may be involved in both affective- and stress-related disorders.

3.7. Dynorphins. The complete sequence of dynorphin A (Dyn-A) (17amino acids) was identified by Goldstein et al. [132] in 1981. The prefix dyn-was taken from the Greek (power) whereas-orphin suggests its opioid nature. DynA might play a regulatory role in epilepsy and addiction, whereas findings related to emotional control are quite conflicting. Data are mainly derived from animal models and a correct interpretation of findings from mouse testing has to be observed in the general context of a different social behavior in rats and mice [133]. Exploring the effects and molecular mechanism of action of dynorphins in humans is a very complicated task considering mood and anxiety behaviors that may be investigated only indirectly through a detailed interpretation of animal models.

3.8. Orexin. Orexins or hypocretins and their receptors have been discovered in 1998 as predominantly expressed in the lateral hypothalamus [134], specifically in the perifornical region of the lateral hypothalamus and the posterior hypothalamic area $[135,136]$. The name hypocretins is related to the similar amino acid sequence of the gut peptide secretin, whereas the name orexins is derived by the Greek word orexis which means appetite (these are peptides that stimulate food intake) [137].

Hypocretin fibers such as the central nucleus of the amygdala (CeA), bed nucleus of the stria terminalis (BNST), $\mathrm{LC}$, and paraventricular nucleus of the hypothalamus (PVN) might have a specific role in the regulationof stress responses [136]. Hypocretins bind the hypocretin-1 and hypocretin-2 receptors. Hypocretin-1 has a 2-3-fold higher affinity for the hypocretin-1 receptor than hypocretin-2 [137]. Several studies have provided evidence for a role of orexins/hypocretins in the regulation of stress responses and in the regulation of many human behaviors.

3.9. The Association between Neuropeptides and Suicidality. Neuropeptides may play a key role in the stress response as well as in the regulation of several human behaviors. Table 2 summarized the most relevant studies addressing the association between neuropeptides and suicide.

Important insight into the role of neuropeptides in the pathogenesis of MDD and suicidal behavior has been given. Orexin alterations have been investigated in several subgroups of patients. In a factor analysis of CSF biomarkers in suicide attempters, a negative loading on orexin, along with positive loading on proinflammatory cytokine interleukin-6 
and monoamine metabolites 5-HIAA and HVA was associated with violent suicide method and risk for future suicide completion [83]. Orexin levels were also reported to be significantly lower in patients with MDD compared to those with adjustment disorder and dysthymia [87]. The same research group has also found that lower orexin levels were associated with depressive symptoms such as higher inertia and decreased motor activity in 101 suicide attempters [88]. As later reported by Brundin et al. [85], a significant negative correlation was found between the change in CSF-orexin levels and symptoms of suicidality, assessed by SUAS [138], one year after a suicide attempt.

Furthermore, Inder et al. [93] found a significant positive correlation between plasma AVP, ACTH, and cortisol levels in depressed subjects. The authors reported that plasma AVP levels were increased in those subjects who had attempted suicide whereas no difference was observed in mean cortisol between depressed subjects and controls.

Patients with NSSI were also investigated in order to find abnormalities in neuropeptides. Stanley et al. [84] recruited 29 psychiatric patients with a history of one or more suicide attempts with a mean of three attempts. Patients with NSSI had lower CSF $\beta$-endorphin levels and met-enkephalin when compared to a diagnostically matched group of patients without NSSI but no difference in dynorphin levels was found between the two groups.

The excessive CRH secretion and neurotransmission which is involved in the pathophysiology of depressive disorders has been suggested to extend beyond the hypothalamus and to involve several extrahypothalamic brain regions. Austin et al. [89] found a significant positive correlation between the magnitude of the increase in CRH-IR levels in the LC and the age at illness onset in a sample of 11 depressed subjects who died by suicide compared with controls. Specifically, the mean level of CRH-IR in depressed subjects was significantly increased by $30 \%$ in the LC, by $45 \%$ in the caudal nucleus of the DR and by $39 \%$ in the median raphe relative to healthy controls.

In addition, Träskman-Bendz et al. [100] reported that, in a sample of 44 suicide attempters, patients who had made previous suicide attempts had significantly lower CRH than those who had not, and only patients with MDD had significantly lower somatostatin, CRH, and DSIP compared to patients with other diagnoses. Westrin et al. [95] found that levels of CSF-somatostatin, but not CSF-CRH, increased with clinical improvement in suicidal patients independently of psychiatric diagnoses.

Westrin et al. [96] found that in a sample of $34 \mathrm{MDD}$ suicidal patients, those with previous suicide attempts had increased plasma DSIP levels. Scarone et al. [101] suggested reduced beta-endorphin levels in the left temporal cortex, frontal cortex, and caudate nucleus in 7 suicides compared to 7 controls. An asymmetrical concentration of beta-endorphins in suicides (left less than right) was also suggested in frontal cortex and caudate nucleus.

Whether psychoactive treatments affect neuropeptides levels is a matter of debate. Olsson et al. [94] found that antidepressant treatment seemed to affect the levels of CSF NPY and SP. Westrin et al. [33] suggested that there may be alterations in CRH and NPY plasma levels in patients with a mood disorder who had recently attempted suicide. Specifically, the authors found that cortisol was significantly high and CRH and NPY were low in recent suicide attempters. Moreover, Westrin et al. [97] reported that NPY correlated positively with psychasthenia, irritability, and stability in patients and negatively with muscular tension, psychasthenia, verbal aggression, and irritability in controls. Cortisol correlated positively with validity, extraversion, and verbal aggression and negatively with inhibition of aggression in controls.

Also, post-mortem studies have been conducted in order to analyze the possible role of neuropeptides in suicidal behavior. Merali et al. [90] suggested that in a sample of 30 suicides and 37 controls, suicides were associated with sitespecific alterations in the endogenous levels of CRH, AVP, GRP, and NMB. However, GRP and NMB variations were limited although GRP-ir within the LC of suicides was higher than in control subjects and NMB-ir which was reduced at the DVC of suicides. In another post-mortem study, Caberlotto and Hurd [92] reported that no significant alterations in $\mathrm{Y} 1$ or Y2 mRNA expression levels were observed between suicides and controls. The Y2 mRNA expression was increased in layer IV of suicides. There was a negative correlation between Y1 mRNA expression levels in the prefrontal cortex and increasing age.

Moreover, Widdowson et al. [34] reported the existence of NPY deficit in the brain leading to region-specific reductions in neuropeptide concentrations in individuals with a history of depression. In this post-mortem study, the authors found that NPY levels were significantly lower in the frontal cortex and caudate nucleus of suicide victims compared to agematched controls.

Negative findings have also been reported. Pitchot et al. [86] found no correlation between severity of depressive symptoms and AVP-neurophysins or post-DST cortisol levels in a sample of $28 \mathrm{MDD}$ inpatients. Any correlation between AVP-neurophysins and post-DST cortisol levels $(r=-0.07$, $P=0.72$ ) was observed. AVP neurophysins did not differ between DST suppressors and nonsuppressors. In addition, Brunner et al. [91] found no differences in CSF AVP concentrations between 19 depressed suicide attempters, depressed nonsuicidal patients, and 9 neurological control subjects and there was no relationship between CSF AVP and monoamine metabolites in CSF.

In a 5-year follow-up study, Roy [98] did not find evidence that NPY, somatostatin, diazepam-binding inhibitor, GABA, or CRH were major determinants of suicidal behavior or its repetition in depression, since no significant differences between depressed patients who did or did not reattempt suicide were found. Also, Ordway et al. [99] found no significant differences in NPY concentrations between suicide victims with MDD (with comorbid alcohol dependence) and agematched control subjects. Finally, Maes et al. [102] reported no significant differences in TSH, FT4, ACTH, and total LTRP levels between patients with suicidal ideation and those without. 


\section{Discussion}

The present review supports a role of neuropeptides in the pathophysiology of suicidal behavior. Based on the included studies, there may be associations between neuropeptides and suicidal behavior but this does not imply the existence of a causal link. Most studies (thirtheen) $[33,34,83-85,89,90,92-$ $96,101]$ included in our review found an association between neuropeptides and suicidal behavior; however, there are also six studies $[86,91,98-100,102]$ that did not confirm this association. Moreover, there are other studies suggesting that neuropeptides abnormalities may be found in subjects with MDD but not suicidal behavior [87, 88, 97].

One relevant limitation of the selected studies is related to the variable neuropeptide concentrations (e.g., some studies reporting increases and others reporting reductions or no significant changes) in those who attempted suicide or died by suicide compared with healthy controls or those dying a sudden natural or accidental death, respectively. These findings reflected the significant heterogeneity in terms of psychopathology which is commonly observed in those with suicidal behavior together with the variability in the antidepressant response which may be found in these different subgroups of patients. Specific stressors may induce specific neuropeptide responses: SP may be evoked by structural damage, oxytocin release by social threats, melanin-concentrating hormone $(\mathrm{MCH})$ by abnormal energy homeostasis, and AVP changes by blood volume changes, respectively. Neuropeptides such as CRF, VGF, CCK, SP, and NPY have been demonstrated to act as key neuromodulators of emotional processing $[139,140]$.

Neuropeptides may be considered as crucial molecules in the interaction between genes and environment as well as fundamental mediators of the stress response able to in turn affect gene regulation in order to respond to the different environmental stimuli. What is the main mechanism underlying neuropeptides alterations in subjects with suicidal behavior? Evidence suggests that modulation of monoaminergic transmission may represent the main mechanism by which the neuropeptide galanin was implicated in stressrelated disorders [103]. This modulation of monoaminergic systems has been observed predominantly in the LC and DR nuclei. At the molecular level, stressful events may enhance the expression of tyrosine hydroxylase [141-143], the enzyme which is involved in controlling catecholamine synthesis, and GAL $[103,144]$ with the final increase of noradrenergic transmission. This may determine both direct and indirect inhibitory effects on postsynaptic neuronal functions implicated in depression-like behaviors.

The role of NPY and its receptors in affective disorders and stress-related conditions has been investigated for over two decades but still remains rather controversial. According to behavioral findings on NPY, Y1, and Y2 receptors in animal models, evidence suggested an important role of NPY in emotional responses and stress- or depression-related disorders [145]. Westrin et al. [33] reported significantly higher cortisol, $\mathrm{CRH}$, and lower NPY levels in recent suicide attempters when compared with healthy controls. NPY was also reported by the same research group to correlate positively with psychasthenia, irritability, and stability and negatively with validity in patients [97]. There are also studies that did not report significant changes in neuropeptides concentrations in patients with suicidal behavior. There were no significant differences in CSF NPY, somatostatin, diazepam-binding inhibitor, GABA, or CRH levels between depressed patients who did or did not reattempt suicide during the follow-up or those who had never attempted according to Roy [98]. However, a nonsuicidal control group was not included in the mentioned study. Moreover, Ordway et al. [99] did not find an association between NPY and completed suicide, although the fact that some suicide victims had a history of comorbid alcohol dependency who may have confounded the results.

Pitchot and colleagues [86] found no association between major depressive symptoms as assessed by HDRS and AVPneurophysins levels. However, the authors of this study stated that plasma AVP levels presumably did not reflect AVP activity in the mangocellular portion of the paraventricular nucleus. Also, no significant difference in dynorphin levels was found between patients with a history of one or more suicide attempts [84]. Here, it is important to note that plasma neuropeptide levels may not accurately reflect central neuropeptide levels. In addition, Westrin et al. [95] found no significant changes in CSF-CRH at follow-up in 16 patients of which eleven had made previous suicide attempts. However, the substances which have been ingested by patients may have caused the initially low CRH and somatostatin levels, and thus confounded the results. Furthermore, this study did not demonstrate whether somatostatin changes are specifically related to either suicidality or depression but only that somatostatin changes are related to clinical symptomatic recovery in suicidal patients.

Finally, based on Maes et al. investigation [102], no relevant (significant) differences were observed in terms of TSH, FT4, pre- and postdexamethasone cortisol, ACTH, and L-TRP levels between patients with suicidal ideation and those without. However, considering the possible caveats, this study was cross-sectional in nature and subjects were not assessed using psychometric instruments.

Many of the mentioned studies should be considered in the light of the following shortcomings. Many studies were not completely informative over time and lacked adequate long-term follow-up periods. The naturalistic designs may not completely control for the effect of confounding factors such as the duration of treatment and disease and the diagnostic subtypes. Also, samples were often too small or included mixed, heterogeneous patients and usually did not allow to generalize findings. Patients with suicidal behavior who were included in these studies may be affected by different psychiatric disorders; the methods of suicide attempts were not controlled in all studies. Differences may exist between participants and those that did not accept to participate in the studies. Furthermore, some of these studies may lack a control group or, alternatively, controls were not matched for age and other clinically relevant information. In addition, psychometric instruments evaluating suicide risk were not used in all studies.

Moreover, many of the observed abnormalities in neuropeptides concentrations were found in subjects with suicidal behavior who were also depressed. It is possible that 
the association between neuropeptides and suicidality may be mediated by the existence of psychiatric conditions such as major affective disorders. Generally, the variability in the concentrations of neuropeptide markers is too great to draw any definitive conclusion in those who were depressed and also displayed suicidality.

In light of recent advances in understanding the regulation of genes encoding neuropeptides, neuropeptide receptors have emerged as attractive targets for the treatment of many psychiatric disorders. To date, many molecules have been synthesized but none has been introduced into the market due to the failure of most clinical trials aimed to show their efficacy. Griebel and Holsboer [146] stated in their review that although no strong mechanistic hypothesis supports the active role of the neuropeptide system in psychiatric conditions, medications targeting neuropeptide receptors have not completely failed to demonstrate their efficacy. They analyzed preclinical and clinical evidence on nonpeptidergic ligands for neuropeptide receptors in psychiatric disorders mainly focusing on tachykinins, CRF, vasopressin, and neurotensin. Overall, the authors suggested the clinical potential of neuropeptide receptor antagonists as candidate medications that may help to reduce the toll of major affective disorders and suicide but reported the importance to stratify patients according to the different subtypes and illness severity. Objectifiable laboratory tests should be available to go beyond the issue of heterogeneity of patients recruited in the different studies. Moreover, the authors recommended the careful knowledge of DNA sequence variations of neuropeptides and they highlighted the importance to ensure that abnormalities in neuropeptide signalling represent one of the most relevant pathogenetic factors in major depression.

Biomarkers and genetic tests are suggested to identify clinical conditions that may be related to a specific neuropeptidergic mechanism. The specificity of the mechanism of action of the different neuropeptides should also be adequately considered. An important research question that needs to be addressed in future studies is how and when personalizing treatments using drugs targeting neuropeptide receptors are possible. This implies the knowledge of whether subjects could preferably respond to a medication or another, which may be the right combination of medications, and what is the real benefit for some subgroups of patients.

The possibility to select medications targeting neuropeptide receptors according to findings derived from animal studies should be carefully considered using models evaluating different aspects of the disorders.

A detailed characterization of patients based on objectifiable measures, genetic tests, and reliable biomarkers is undoubtedly requested.

\section{Conclusion}

According to most of the studies included in the present review, neuropeptides may play a key role in the pathophysiology of suicidal behavior. In particular, one mechanism by which neuropeptide alterations may promote suicidal symptoms is via changes in monoaminergic systems rendering neuropeptides as novel, intriguing mediators of stress-related and affective conditions as well as suicidality.

However, despite the recent advances in this research field, it is unlikely that neuropeptides represent definitive and reliable markers of suicidality. Further additional studies will elucidate the pathophysiological mechanisms of the different neuropeptides pathways underlying suicidal behavior.

\section{References}

[1] S. K. Goldsmith, T. C. Pellmar, A. M. Kleinman, and W. E. Bunney, Reducing Suicide, Institute of Medicine of the US National Academies of Science, Washington, DC, USA, 2002.

[2] R. I. Simon and R. E. Hales, Textbook of Suicide Assessment and Management, American Psychiatric Press, Washington, DC, USA, 2006.

[3] World Health Organization (WHO), "International suicide rates," http://www.who.int/mental_health/prevention/suicide/ country_reports/en/index.html.

[4] L. Tondo, G. Isacsson, and R. J. Baldessarini, "Suicidal behaviour in bipolar disorder: risk and prevention," CNS Drugs, vol. 17, no. 7, pp. 491-511, 2003.

[5] R. C. Kessler, P. Berglund, G. Borges, M. Nock, and P. S. Wang, "Trends in suicide ideation, plans, gestures, and attempts in the United States, 1990-1992 to 2001-2003," Journal of the American Medical Association, vol. 293, no. 20, pp. 2487-2495, 2005.

[6] A. Roy, "Suicide," in Kaplan and Sadock's Comprehensive Textbook of Psychaitry, B. J. Sadock and V. A. Sadock, Eds., vol. 2, pp. 2031-2040, Lippincott Willians and Wilkins, Philadelphia, $\mathrm{Pa}, \mathrm{USA}, 7$ th edition, 2000.

[7] M. Pompili, M. Innamorati, Z. Rihmer et al., "Cyclothymicdepressive-anxious temperament pattern is related to suicide risk in 346 patients with major mood disorders," Journal of Affective Disorders, vol. 136, no. 3, pp. 405-411, 2012.

[8] M. Pompili, Z. Rihmer, H. Akiskal et al., "Temperaments mediate suicide risk and psychopathology among patients with bipolar disorders," Comprehensive Psychiatry, vol. 53, no. 3, pp. 280-285, 2012.

[9] M. Pompili, G. Serafini, M. Innamorati et al., "Suicide risk in first episode psychosis: a selective review of the current literature," Schizophrenia Research, vol. 129, no. 1, pp. 1-11, 2011.

[10] G. Serafini, M. Pompili, M. Innamorati et al., "Affective temperamental profiles are associated with white matter hyperintensity and suicidal risk in patients with mood disorders," Journal of Affective Disorders, vol. 129, no. 1-3, pp. 47-55, 2011.

[11] G. Serafini, M. Pompili, M. Innamorati et al., "The role of microRNAs in synaptic plasticity, major affective disorders and suicidal behavior," Neuroscience Research, vol. 73, pp. 179-190, 2012.

[12] M. Innamorati, M. Pompili, X. Gonda et al., "Psychometric properties of the Gotland Scale for Depression in Italian psychiatric inpatients and its utility in the prediction of suicide risk," Journal of Affective Disorders, vol. 132, no. 1-2, pp. 99-103, 2011.

[13] R. W. Maris, “Suicide," The Lancet, vol. 360, no. 9329, pp. 319326, 2002.

[14] K. Hawton and K. van Heeringen, "Suicide," The Lancet, vol. 373, no. 9672, pp. 1372-1381, 2009.

[15] E. Baca-Garcia, C. Diaz-Sastre, E. García Resa et al., "Suicide attempts and impulsivity," European Archives of Psychiatry \& Clinical Neuroscience, vol. 255, no. 2, pp. 152-156, 2005. 
[16] Y. Gvion and A. Apte, "Aggression, impulsivity, and suicide behavior: a review of the literature," Archives of Suicide Research, vol. 15, no. 2, pp. 93-112, 2011.

[17] E. Corruble, A. Benyamina, F. Bayle, B. Falissard, and P. Hardy, "Understanding impulsivity in severe depression? A psychometrical contribution," Progress in Neuro-Psychopharmacology \& Biological Psychiatry, vol. 27, no. 5, pp. 829-833, 2003.

[18] R. Plutchik and H. M. Van Praag, "The measurement of suicidality, aggressivity and impulsivity," Clinical Neuropharmacology, vol. 9, supplement 4, pp. S380-S382, 1986.

[19] A. T. Beck, I. Herman, and D. Schuyler, "Development of suicidal intent scales," in The Prediction of Suicide, A. T. Beck, H. L. P. Resnick, and D. Lettieri, Eds., pp. 45-46, Charles Press, 1974.

[20] P. McGonigle, "Peptide therapeutics for CNS indications," Biochemical Pharmacology, vol. 83, no. 5, pp. 559-566, 2012.

[21] M. E. Keck, F. Ohl, F. Holsboer, and M. B. Müller, "Listening to mutant mice: a spotlight on the role of CRF/CRF receptor systems in affective disorders," Neuroscience \& Biobehavioral Reviews, vol. 29, no. 4-5, pp. 867-889, 2005.

[22] J. Panksepp, “The neurochemistry of behavior," Annual Review of Psychology, vol. 37, pp. 77-107, 1986.

[23] B. Alldredge, "Pathogenic involvement of neuropeptides in anxiety and depression," Neuropeptides, vol. 44, no. 3, pp. 215224,2010

[24] M. Ludwig, "Are neuropeptides brain hormones?" Journal of Neuroendocrinology, vol. 23, no. 4, pp. 381-382, 2011.

[25] T. Hökfelt, C. Broberger, Z.-Q. D. Xu, V. Sergeyev, R. Ubink, and M. Diez, "Neuropeptides-an overview," Neuropharmacology, vol. 39, no. 8, pp. 1337-1356, 2000.

[26] J. Harro, "CCK and NPY as anti-anxiety treatment targets: promises, pitfalls, and strategies," Amino Acids, vol. 31, no. 3, pp. 215-230, 2006.

[27] R. J. Valentino and K. G. Commons, "Peptides that fine-tune the serotonin system," Neuropeptides, vol. 39, no. 1, pp. 1-8, 2005.

[28] P. W. Gold, G. Chrousos, C. Kellner et al., "Psychiatric implications of basic and clinical studies with corticotropin-releasing factor," American Journal of Psychiatry, vol. 141, no. 5, pp. 619627, 1984.

[29] F. Holsboer, "The corticosteroid receptor hypothesis of depression," Neuropsychopharmacology, vol. 23, no. 5, pp. 477-501, 2000.

[30] M. E. Keck, T. Welt, M. B. Müller et al., "Reduction of hypothalamic vasopressinergic hyperdrive contributes to clinically relevant behavioral and neuroendocrine effects of chronic paroxetine treatment in a psychopathological rat model," Neuropsychopharmacology, vol. 28, no. 2, pp. 235-243, 2003.

[31] T. Sherrin, K. Y. C. Heng, Y. Z. Zhu, Y. M. Tang, G. Lau, and C. H. Tan, "Cholecystokinin-B receptor gene expression in cerebellum, pre-frontal cortex and cingulate gyrus and its association with suicide," Neuroscience Letters, vol. 357, no. 2, pp. 107-110, 2004.

[32] J. Harro, J. Marcusson, and L. Oreland, "Alterations in brain cholecystokinin receptors in suicide victims," European Neuropsychopharmacology, vol. 2, no. 1, pp. 57-63, 1992.

[33] Å. Westrin, R. Ekman, and L. Träskman-Bendz, "Alterations of corticotropin releasing hormone $(\mathrm{CRH})$ and neuropeptide $\mathrm{Y}$ (NPY) plasma levels in mood disorder patients with a recent suicide attempt," European Neuropsychopharmacology, vol. 9, no. 3, pp. 205-211, 1999.

[34] P. S. Widdowson, G. A. Ordway, and A. E. Halaris, "Reduced neuropeptide Y concentrations in suicide brain," Journal of Neurochemistry, vol. 59, no. 1, pp. 73-80, 1992.
[35] M. Heilig, "Neuropeptide Y in relation to behaviour and psychiatric disorders," in Biology of Neuropeptide Y and Related Peptides, W. F. Colmers and C. Wahlestedt, Eds., pp. 511-554, Humana Press, Totowa, NJ, USA, 1993.

[36] D. Moher, D. J. Cook, S. Eastwood, I. Olkin, D. Rennie, and D. F. Stroup, "Improving the quality of reports of meta-analyses of randomised controlled trials: the QUOROM statement. QUOROM Group," The British Journal of Surgery, vol. 87, no. 11, pp. 1448-1454, 2000.

[37] S. C. Heinrichs and G. F. Koob, "Corticotropin-releasing factor in brain: a role in activation, arousal, and affect regulation," Journal of Pharmacology and Experimental Therapeutics, vol. 311, no. 2, pp. 427-440, 2004.

[38] C. B. Nemeroff and W. W. Vale, "The neurobiology of depression: inroads to treatment and new drug discovery," Journal of Clinical Psychiatry, vol. 66, supplement 7, pp. 5-13, 2005.

[39] J. H. Kehne, "The CRF1 receptor, a novel target for the treatment of depression, anxiety, and stress-related disorders," CNS \& Neurological Disorders-Drug Targets, vol. 6, no. 3, pp. 163-182, 2007.

[40] M. Pompili, G. Serafini, M. Innamorati et al., "The hypothalamic-pituitary-adrenal axis and serotonin abnormalities: a selective overview for the implications of suicide prevention," European Archives of Psychiatry \& Clinical Neuroscience, vol. 260, no. 8, pp. 583-600, 2010.

[41] C. B. Nemeroff, G. Bissette, H. Akil, and M. Fink, "Neuropeptide concentrations in the cerebrospinal fluid of depressed patients treated with electroconvulsive therapy. Corticotrophin-releasing factor, $\beta$-endorphin and somatostatin," British Journal of Psychiatry, vol. 158, pp. 59-63, 1991.

[42] S. C. Stout, M. J. Owens, and C. B. Nemeroff, "Regulation of corticotropin-releasing factor neuronal systems and hypothalamic-pituitary-adrenal axis activity by stress and chronic antidepressant treatment," Journal of Pharmacology and Experimental Therapeutics, vol. 300, no. 3, pp. 1085-1092, 2002.

[43] F. Holsboer, "The corticosteroid receptor hypothesis of depression," Neuropsychopharmacology, vol. 23, no. 5, pp. 477-501, 2000.

[44] C. B. Nemeroff, E. Widerlov, and G. Bissette, "Elevated concentrations of CSF corticotropin-releasing factor-like immunoreactivity in depressed patients," Science, vol. 226, no. 4680, pp. 1342-1344, 1984.

[45] G. Bissette, V. Klimek, J. Pan, C. Stockmeier, and G. Ordway, "Elevated concentrations of CRF in the locus coeruleus of depressed subjects," Neuropsychopharmacology, vol. 28, no. 7, pp. 1328-1335, 2003.

[46] Z. Merali, L. Du, P. Hrdina et al., "Dysregulation in the suicide brain: mRNA expression of corticotropinreleasing hormone receptors and GABA(A) receptor subunits in frontal cortical brain region," Journal of Neuroscience, vol. 24, no. 6, pp. 14781485, 2004.

[47] C. B. Nemeroff, M. J. Owens, G. Bissette, A. C. Andorn, and M. Stanley, "Reduced corticotropin releasing factor binding sites in the frontral cortex of suicide victims," Archives of General Psychiatry, vol. 45, no. 6, pp. 577-579, 1988.

[48] S. Makino, M. A. Smith, and P. W. Gold, "Increased expression of corticotropin-releasing hormone and vasopressin messenger ribonucleic acid (mRNA) in the hypothalamic paraventricular nucleus during repeated stress: association with reduction in glucocorticoid receptor mRNA levels," Endocrinology, vol. 136, no. 8, pp. 3299-3309, 1995. 
[49] G. L. Forster, R. B. Pringle, N. J. Mouw et al., "Corticotropinreleasing factor in the dorsal raphe nucleus increases medial prefrontal cortical serotonin via type 2 receptors and median raphe nucleus activity," European Journal of Neuroscience, vol. 28, no. 2, pp. 299-310, 2008.

[50] Z. Liu, F. Zhu, G. Wang et al., "Association of corticotropinreleasing hormone receptorl gene SNP and haplotype with major depression," Neuroscience Letters, vol. 404, no. 3, pp. 358$362,2006$.

[51] Z. Liu, F. Zhu, G. Wang et al., "Association study of corticotropin-releasing hormone receptorl gene polymorphisms and antidepressant response in major depressive disorders," Neuroscience Letters, vol. 414, no. 2, pp. 155-158, 2007.

[52] M. E. Keck, N. Kern, A. Erhardt et al., "Combined effects of exonic polymorphisms in CRHR1 and AVPR1B genes in a case/control study for panic disorder," American Journal of Medical Genetics B, vol. 147, no. 7, pp. 1196-1204, 2008.

[53] R. G. Bradley, E. B. Binder, M. P. Epstein et al., "Influence of child abuse on adult depression: moderation by the corticotropin-releasing hormone receptor gene," Archives of General Psychiatry, vol. 65, no. 2, pp. 190-200, 2008.

[54] B. Binneman, D. Feltner, S. Kolluri, Y. Shi, R. Qiu, and T. Stiger, "A 6-week randomized, placebo-controlled trial of CP316,311 (a selective CRH1 antagonist) in the treatment of major depression," American Journal of Psychiatry, vol. 165, no. 5, pp. 617-620, 2008.

[55] H. K. Caldwell, H.-J. Lee, A. H. Macbeth, and W. S. Young III, "Vasopressin: behavioral roles of an "original" neuropeptide," Progress in Neurobiology, vol. 84, no. 1, pp. 1-24, 2008.

[56] L. van Londen, J. G. Goekoop, G. M. J. van Kempen et al., "Plasma levels of arginine vasopressin elevated in patients with major depression," Neuropsychopharmacology, vol. 17, no. 4, pp. 284-292, 1997.

[57] J. S. Purba, W. J. G. Hoogendijk, M. A. Hofman, and D. F. Swaab, "Increased number of vasopressin- and oxytocin-expressing neurons in the paraventricular nucleus of the hypothalamus in depression," Archives of General Psychiatry, vol. 53, no. 2, pp. 137-143, 1996.

[58] E. D. Schmidt, R. Binnekade, A. W. J. W. Janszen, and F. J. H. Tilders, "Short stressor induced long-lasting increases in vasopressin stores in hypothalamic corticotropin-releasing hormone (CRH) neurons in adult rats," Journal of Neuroendocrinology, vol. 8, no. 9, pp. 703-712, 1996.

[59] S. Volpi, C. Rabadan-Diehl, and G. Aguilera, "Vasopressinergic regulation of the hypothalamic pituitary adrenal axis and stress adaptation," Stress, vol. 7, no. 2, pp. 75-83, 2004.

[60] T. G. Dinan and L. V. Scott, "Anatomy of melancholia: focus on hypothalamic-pituitary-adrenal axis overactivity and the role of vasopressin," Journal of Anatomy, vol. 207, no. 3, pp. 259-264, 2005.

[61] E. L. Dempster, I. Burcescu, K. Wigg et al., "Evidence of an association between the vasopressin Vlb receptor gene (AVPR1B) and childhood-onset mood disorders," Archives of General Psychiatry, vol. 64, no. 10, pp. 1189-1195, 2007.

[62] N. Egashira, A. Tanoue, T. Matsuda et al., "Impaired social interaction and reduced anxiety-related behavior in vasopressin Vla receptor knockout mice," Behavioural Brain Research, vol. 178, no. 1, pp. 123-127, 2007.

[63] C. J. Bleickardt, D. E. Mullins, C. P. MacSweeney et al., "Characterization of the Vla antagonist, JNJ-17308616, in rodent models of anxiety-like behavior," Psychopharmacology, vol. 202, no. 4, pp. 711-718, 2009.
[64] G. Aguilera, S. Subburaju, S. Young, and J. Chen, "The parvocellular vasopressinergic system and responsiveness of the hypothalamic pituitary adrenal axis during chronic stress," Progress in Brain Research, vol. 170, pp. 29-39, 2008.

[65] N. G. Simon, C. Guillon, K. Fabio et al., "Vasopressin antagonists as anxiolytics and antidepressants: recent developments," Recent Patents on CNS Drug Discovery, vol. 3, no. 2, pp. 77-93, 2008.

[66] C. A. Pedersen and M. L. Boccia, "Oxytocin antagonism alters rat dams' oral grooming and upright posturing over pups," Physiology \& Behavior, vol. 80, no. 2-3, pp. 233-241, 2003.

[67] A. Benelli, A. Bertolini, R. Poggioli, B. Menozzi, R. Basaglia, and R. Arletti, "Polymodal dose-response curve for oxytocin in the social recognition test," Neuropeptides, vol. 28, no. 4, pp. 251255, 1995.

[68] U. Rimmele, K. Hediger, M. Heinrichs, and P. Klaver, "Oxytocin makes a face in memory familiar," Journal of Neuroscience, vol. 29, no. 1, pp. 38-42, 2009.

[69] A. Stachowiak, C. Macchi, G. G. Nussdorfer, and L. K. Malendowicz, "Effects of oxytocin on the function and morphology of the rat adrenal cortex: in vitro and in vivo investigations," Research in Experimental Medicine, vol. 195, no. 5, pp. 265-274, 1995.

[70] J.-J. Legros, "Inhibitory effect of oxytocin on corticotrope function in humans: are vasopressin and oxytocin ying-yang neurohormones?" Psychoneuroendocrinology, vol. 26, no. 7, pp. 649-655, 2001.

[71] R. H. Ring, J. E. Malberg, L. Potestio et al., "Anxiolytic-like activity of oxytocin in male mice: behavioral and autonomic evidence, therapeutic implications," Psychopharmacology, vol. 185, no. 2, pp. 218-225, 2006.

[72] R. H. Ring, L. E. Schechter, S. K. Leonard et al., "Receptor and behavioral pharmacology of WAY-267464, a non-peptide oxytocin receptor agonist," Neuropharmacology, vol. 58, no. 1, pp. 69-77, 2010.

[73] P. Kirsch, C. Esslinger, Q. Chen et al., "Oxytocin modulates neural circuitry for social cognition and fear in humans," Journal of Neuroscience, vol. 25, no. 49, pp. 11489-11493, 2005.

[74] G. Scantamburlo, M. Hansenne, S. Fuchs et al., "Plasma oxytocin levels and anxiety in patients with major depression," Psychoneuroendocrinology, vol. 32, no. 4, pp. 407-410, 2007.

[75] K. Uvnas-Moberg, P. Alster, and T. H. Svensson, "Amperozide and clozapine but not haloperidol or raclopride increase the secretion of oxytocin in rats," Psychopharmacology, vol. 109, no. 4, pp. 473-476, 1992.

[76] J. Jokinen, A. Chatzittofis, C. Hellström, P. Nordström, K. Uvnäs-Moberg, and M. Åsberg, "Low CSF oxytocin reflects high intent in suicide attempters," Psychoneuroendocrinology, vol. 37, no. 4, pp. 482-490, 2012.

[77] D. Feifel and T. Reza, "Oxytocin modulates psychotomimeticinduced deficits in sensorimotor gating," Psychopharmacology, vol. 141, no. 1, pp. 93-98, 1999.

[78] D. Feifel, K. MacDonald, A. Nguyen et al., "Adjunctive intranasal oxytocin reduces symptoms in schizophrenia patients," Biological Psychiatry, vol. 68, no. 7, pp. 678-680, 2010.

[79] C. Modahl, L. Green, D. Fein et al., "Plasma oxytocin levels in autistic children," Biological Psychiatry, vol. 43, no. 4, pp. 270277, 1998.

[80] C. M. Yrigollen, S. S. Han, A. Kochetkova et al., "Genes controlling affiliative behavior as candidate genes for autism," Biological Psychiatry, vol. 63, no. 10, pp. 911-916, 2008. 
[81] E. Hollander, J. Bartz, W. Chaplin et al., "Oxytocin increases retention of social cognition in autism," Biological Psychiatry, vol. 61, no. 4, pp. 498-503, 2007.

[82] E. Hollander, S. Novotny, M. Hanratty et al., "Oxytocin infusion reduces repetitive behaviors in adults with autistic and Asperger's disorders," Neuropsychopharmacology, vol. 28, no. 1, pp. 193-198, 2003.

[83] D. Lindqvist, S. Janelidze, S. Erhardt, L. Träskman-Bendz, G. Engström, and L. Brundin, "CSF biomarkers in suicide attempters-a principal component analysis," Acta Psychiatrica Scandinavica, vol. 124, no. 1, pp. 52-61, 2011.

[84] B. Stanley, L. Sher, S. Wilson, R. Ekman, Y.-Y. Huang, and J. J. Mann, "Non-suicidal self-injurious behavior, endogenous opioids and monoamine neurotransmitters," Journal of Affective Disorders, vol. 124, no. 1-2, pp. 134-140, 2010.

[85] L. Brundin, M. Björkqvist, L. Träskman-Bendz, and A. Petersén, "Increased orexin levels in the cerebrospinal fluid the first year after a suicide attempt," Journal of Affective Disorders, vol. 113, pp. 179-182, 2009.

[86] W. Pitchot, G. Scantamburlo, E. Pinto et al., "Vasopressinneurophysin and DST in major depression: relationship with suicidal behavior," Journal of Psychiatric Research, vol. 42, no. 8, pp. 684-688, 2008.

[87] L. Brundin, M. Björkqvist, Å. Petersén, and L. TräskmanBendz, "Reduced orexin levels in the cerebrospinal fluid of suicidal patients with major depressive disorder," European Neuropsychopharmacology, vol. 17, no. 9, pp. 573-579, 2007.

[88] L. Brundin, A. Petersén, M. Bjirkqvist, and L. Träskman-Bendz, "Orexin and psychiatric symptoms in suicide attempters," Journal of Affective Disorders, vol. 100, no. 1-3, pp. 259-263, 2007.

[89] M. C. Austin, J. E. Janosky, and H. A. Murphy, "Increased corticotropin-releasing hormone immunoreactivity in monoamine-containing pontine nuclei of depressed suicide men," Molecular Psychiatry, vol. 8, no. 3, pp. 324-332, 2003.

[90] Z. Merali, P. Kent, L. Du et al., "Corticotropin-releasing hormone, arginine vasopressin, gastrin-releasing peptide, and neuromedin B alterations in stress-relevant brain regions of suicides and control subjects," Biological Psychiatry, vol. 59, no. 7, pp. 594-602, 2006.

[91] J. Brunner, M. E. Keck, R. Landgraf, M. Uhr, C. Namendorf, and T. Bronisch, "Vasopressin in CSF and plasma in depressed suicide attempters: preliminary results," European Neuropsychopharmacology, vol. 12, no. 5, pp. 489-494, 2002.

[92] L. Caberlotto and Y. L. Hurd, "Neuropeptide Y Y1 and Y2 receptor mRNA expression in the prefrontal cortex of psychiatric subjects: relationship of Y2 subtype to suicidal behavior," Neuropsychopharmacology, vol. 25, no. 1, pp. 91-97, 2001.

[93] W. J. Inder, R. A. Donald, T. C. R. Prickett et al., "Arginine vasopressin is associated with hypercortisolemia and suicide attempts in depression," Biological Psychiatry, vol. 42, no. 8, pp. 744-747, 1997.

[94] A. Olsson, G. Regnéll, L. Träskman-Bendz, R. Ekman, and Å. Westrin, "Cerebrospinal neuropeptide $\mathrm{Y}$ and substance $\mathrm{P}$ in suicide attempters during long-term antidepressant treatment," European Neuropsychopharmacology, vol. 14, no. 6, pp. 479-485, 2004.

[95] A. Westrin, R. Ekman, G. Regnéll, and L. Träskman-Bendz, "A follow up study of suicide attempters: increase of CSFsomatostatin but no change in CSF-CRH," European Neuropsychopharmacology, vol. 11, no. 2, pp. 135-143, 2001.

[96] Å. Westrin, R. Ekman, and L. Träskman-Bendz, "High delta sleep-inducing peptide-like immunoreactivity in plasma in suicidal patients with major depressive disorder," Biological Psychiatry, vol. 43, no. 10, pp. 734-739, 1998.

[97] Å. Westrin, G. Engstöm, R. Ekman, and L. Träskman-Bendz, "Correlations between plasma-neuropeptides and temperament dimensions differ between suicidal patients and healthy controls," Journal of Affective Disorders, vol. 49, no. 1, pp. 45-54, 1998.

[98] A. Roy, "Neuropeptides in relation to suicidal behavior in depression," Neuropsychobiology, vol. 28, no. 4, pp. 184-186, 1993.

[99] G. A. Ordway, C. A. Stockmeier, H. Y. Meltzer, J. C. Overholser, S. Jaconetta, and P. S. Widdowson, "Neuropeptide Y in frontal cortex is not altered in major depression," Journal of Neurochemistry, vol. 65, no. 4, pp. 1646-1650, 1995.

[100] L. Träskman-Bendz, R. Ekman, G. Regnéll, and R. Öhman, "HPA-related CSF neuropeptides in suicide attempters," European Neuropsychopharmacology, vol. 2, no. 2, pp. 99-106, 1992.

[101] S. Scarone, O. Gambini, G. Calabrese et al., "Asymmetrical distribution of beta-endorphin in cerebral hemispheres of suicides: preliminary data," Psychiatry Research, vol. 32, no. 2, pp. 159-166, 1990.

[102] M. Maes, M. Vandewoude, C. Schotte et al., "Hypothalamicpituitary-adrenal and -thyroid axis dysfunctions and decrements in the availability of L-tryptophan as biological markers of suicidal ideation in major depressed females," Acta Psychiatrica Scandinavica, vol. 80, no. 1, pp. 13-17, 1989.

[103] E. Kuteeva, T. Hökfelt, T. Wardi, and S. O. Ogren, "Galanin, galanin receptor subtypes and depression-like behaviour," Cellular and Molecular Life Sciences, vol. 65, no. 12, pp. 1854-1863, 2008.

[104] R.-M. Karlsson and A. Holmes, "Galanin as a modulator of anxiety and depression and a therapeutic target for affective disease," Amino Acids, vol. 31, no. 3, pp. 231-239, 2006.

[105] C. Tortorella, G. Neri, and G. G. Nussdorfer, "Galanin in the regulation of the hypothalamic-pituitary-adrenal axis (review)," International Journal of Molecular Medicine, vol. 19, no. 4, pp. 639-647, 2007.

[106] S. O. Ogren, E. Kuteeva, T. Hökfelt, and J. Kehr, "Galanin receptor antagonists: a potential novel pharmacological treatment for mood disorders," CNS Drugs, vol. 20, no. 8, pp. 633-654, 2006.

[107] J. M. Weiss, K. A. Boss-Williams, J. P. Moore, M. K. Demetrikopoulos, J. C. Ritchie, and C. H. K. West, “Testing the hypothesis that locus coeruleus hyperactivity produces depressionrelated changes via galanin," Neuropeptides, vol. 39, no. 3, pp. 281-287, 2005.

[108] S. O. Ogren, H. Razani, E. Elvander-Tottie, and J. Kehr, "The neuropeptide galanin as an in vivo modulator of brain 5-HT1A receptors: possible relevance for affective disorders," Physiology \& Behavior, vol. 92, no. 1-2, pp. 172-179, 2007.

[109] C. J. Swanson, T. P. Blackburn, X. Zhang et al., "Anxiolyticand antidepressant-like profiles of the galanin-3 receptor $(\mathrm{Gal} 3)$ antagonists SNAP 37889 and SNAP 398299," Proceedings of the National Academy of Sciences of the United States of America, vol. 102, no. 48, pp. 17489-17494, 2005.

[110] C. R. Elliott-Hunt, R. J. P. Pope, P. Vanderplank, and D. Wynick, "Activation of the galanin receptor 2 (GalR2) protects the hippocampus from neuronal damage," Journal of Neurochemistry, vol. 100, no. 3, pp. 780-789, 2007.

[111] K. Ebner, P. Muigg, G. Singewald, and N. Singewald, "Substance $P$ in stress and anxiety: NK-1 receptor antagonism interacts with key brain areas of the stress circuitry," Annals of the New York Academy of Sciences, vol. 1144, pp. 61-73, 2008. 
[112] P. Blier, G. Gobbi, N. Haddjeri, L. Santarelli, G. Mathew, and R. Hen, "Impact of substance P receptor antagonism on the serotonin and norepinephrine systems: relevance to the antidepressant/anxiolytic response," Journal of Psychiatry and Neuroscience, vol. 29, no. 3, pp. 208-218, 2004.

[113] Q.-P. Ma and C. Bleasdale, "Modulation of brain stem monoamines and $\gamma$-aminobutyric acid by NKI receptors in rats," NeuroReport, vol. 13, no. 14, pp. 1809-1812, 2002.

[114] K. Ebner and N. Singewald, "Stress-induced release of substance $\mathrm{P}$ in the locus coeruleus modulates cortical noradrenaline release," Naunyn-Schmiedeberg's Archives of Pharmacology, vol. 376, no. 1-2, pp. 73-82, 2007.

[115] K. Ebner, N. M. Rupniak, A. Saria, and N. Singewald, "Substance $\mathrm{P}$ in the medial amygdala: emotional stress-sensitive release and modulation of anxiety-related behavior in rats," Proceedings of the National Academy of Sciences of the United States of America, vol. 101, no. 12, pp. 4280-4285, 2004.

[116] K. Ebner, S. B. Sartori, and N. Singewald, "Tachykinin receptors as therapeutic targets in stress-related disorders," Current Pharmaceutical Design, vol. 15, no. 14, pp. 1647-1674, 2009.

[117] T. Furmark, L. Appel, Å. Michelgård et al., “Cerebral blood flow changes after treatment of social phobia with the neurokinin-1 antagonist GR205171, citalopram, or placebo," Biological Psychiatry, vol. 58, no. 2, pp. 132-142, 2005.

[118] C. Louis, J. Stemmelin, D. Boulay, O. Bergis, C. Cohen, and G. Griebel, "Additional evidence for anxiolytic- and antidepressant-like activities of saredutant (SR48968), an antagonist at the neurokinin-2 receptor in various rodent-models," Pharmacology Biochemistry and Behavior, vol. 89, no. 1, pp. 36-45, 2008.

[119] P. W. Smith and L. A. Dawson, "Neurokinin 3 (NK3) receptor modulators for the treatment of psychiatric disorders," Recent Patents on CNS Drug Discovery, vol. 3, no. 1, pp. 1-15, 2008.

[120] P. E. Sawchenko, L. W. Swanson, and R. Grzanna, "Colocalization of neuropeptide $\mathrm{Y}$ immunoreactivity in brainstem catecholaminergic neurons that project to the paraventricular nucleus of the hypothalamus," Journal of Comparative Neurology, vol. 241, no. 2, pp. 138-153, 1985.

[121] J. P. Redrobe, Y. Dumont, A. Fournier, G. B. Baker, and R. Quirion, "Role of serotonin (5-HT) in the antidepressant-like properties of neuropeptide Y (NPY) in the mouse forced swim test," Peptides, vol. 26, no. 8, pp. 1394-1400, 2005.

[122] M. Heilig, B. Soderpalm, J. A. Engel, and E. Widerlov, "Centrally administered neuropeptide Y (NPY) produces anxiolytic-like effects in animal anxiety models," Psychopharmacology, vol. 98, no. 4, pp. 524-529, 1989.

[123] A. Kask, J. Harro, S. von Hörsten, J. P. Redrobe, Y. Dumont, and R. Quirion, "The neurocircuitry and receptor subtypes mediating anxiolytic-like effects of neuropeptide Y," Neuroscience \& Biobehavioral Reviews, vol. 26, no. 3, pp. 259-283, 2002.

[124] M. Nakajima, A. Inui, A. Asakawa et al., "Neuropeptide Y produces anxiety via Y2-type receptors," Peptides, vol. 19, no. 2, pp. 359-363, 1998.

[125] G. Sørensen, C. Lindberg, G. Wörtwein, T. G. Bolwig, and D. P. D. Woldbye, "Differential roles for neuropeptide Y Y1 and Y5 receptors in anxiety and sedation," Journal of Neuroscience Research, vol. 77, no. 5, pp. 723-729, 2004.

[126] M. Heilig, "The NPY system in stress, anxiety and depression," Neuropeptides, vol. 38, no. 4, pp. 213-224, 2004.
[127] T. J. Sajdyk, S. D. Fitz, and A. Shekhar, “The role of neuropeptide $\mathrm{Y}$ in the amygdala on corticotropin-releasing factor receptormediated behavioral stress responses in the rat," Stress, vol. 9, no. 1, pp. 21-28, 2006.

[128] K. Eaton, F. R. Sallee, and R. Sah, "Relevance of neuropeptide Y (NPY) in psychiatry," Current Topics in Medicinal Chemistry, vol. 7, no. 17, pp. 1645-1659, 2007.

[129] N. Lindefors, A. Linden, S. Brene, G. Sedvall, and H. Persson, "CCK peptides and mRNA in the human brain," Progress in Neurobiology, vol. 40, no. 6, pp. 671-690, 1993.

[130] F. J. Vaccarino and J. Rankin, "Nucleus accumbens cholecystokinin (CCK) can either attenuate or potentiate amphetamineinduced locomotor activity: evidence for rostral-caudal differences in accumbens CCK function," Behavioral Neuroscience, vol. 103, no. 4, pp. 831-836, 1989.

[131] J. N. Crawley, "Subtype-selective cholecystokinin receptor antagonists block cholecystokinin modulation of dopaminemediated behaviors in the rat mesolimbic pathway," Journal of Neuroscience, vol. 12, no. 9, pp. 3380-3391, 1992.

[132] A. Goldstein, W. Fischli, L. I. Lowney, M. Hunkapiller, and L. Hood, "Porcine pituitary dynorphin: complete amino acid sequence of the biologically active heptadecapeptide," Proceedings of the National Academy of Sciences of the United States of America, vol. 78, no. 11, pp. 7219-7223, 1981.

[133] K. M. Hegadoren, T. O’Donnell, R. Lanius, N. J. Coupland, and N. Lacaze-Masmonteil, "The role of $\beta$-endorphin in the pathophysiology of major depression," Neuropeptides, vol. 43, no. 5, pp. 341-353, 2009.

[134] L. De Lecea, T. S. Kilduff, C. Peyron et al., "The hypocretins: hypothalamus-specific peptides with neuroexcitatory activity," Proceedings of the National Academy of Sciences of the United States of America, vol. 95, no. 1, pp. 322-327, 1998.

[135] Y. Date, Y. Ueta, H. Yamashita et al., "Orexins, orexigenic hypothalamic peptides, interact with autonomic, neuroendocrine and neuroregulatory systems," Proceedings of the National Academy of Sciences of the United States of America, vol. 96, no. 2, pp. 748-753, 1999.

[136] T. Nambu, T. Sakurai, K. Mizukami, Y. Hosoya, M. Yanagisawa, and K. Goto, "Distribution of orexin neurons in the adult rat brain," Brain Research, vol. 827, no. 1-2, pp. 243-260, 1999.

[137] T. Sakurai, A. Amemiya, M. Ishii et al., "Orexins and orexin receptors: a family of hypothalamic neuropeptides and $G$ protein-coupled receptors that regulate feeding behavior," Cell, vol. 92, no. 4, pp. 573-585, 1998.

[138] B. Stanley, L. Traskman-Bendz, and M. Stanley, "The suicide assessment scale: a scale evaluating change in suicidal behavior," Psychopharmacology Bulletin, vol. 22, no. 1, pp. 200-205, 1986.

[139] Y. Dumont, J. C. Morales-Medina, and R. Quirion, "Neuropeptide $\mathrm{Y}$ and its role in anxiety-related disorders," in Transmitters and Modulators In Health and Disease, S. Shioda, I. Homma, and N. Kato, Eds., pp. 51-82, Springer, Kato Bunmeisha, 2009.

[140] S. Thakker-Varia and J. Alder, "Neuropeptides in depression: role of VGF," Behavioural Brain Research, vol. 197, no. 2, pp. 262 278, 2009.

[141] E. Kuteeva, T. Wardi, L. Lundström et al., "Differential role of galanin receptors in the regulation of depression-like behavior and monoamine/stress-related genes at the cell body level," Neuropsychopharmacology, vol. 33, no. 11, pp. 2573-2585, 2008.

[142] N. F. Biguet, M. Buda, A. Lamouroux, D. Samolyk, and J. Mallet, "Time course of the changes of TH mRNA in rat brain and adrenal medulla after a single injection of reserpine," The EMBO Journal, vol. 5, no. 2, pp. 287-291, 1986. 
[143] A. Berod, N. F. Biguet, S. Dumas, B. Bloch, and J. Mallet, "Modulation of tyrosine hydroxylase gene expression in the central nervous system visualized by in situ hybridization," Proceedings of the National Academy of Sciences of the United States of America, vol. 84, no. 6, pp. 1699-1703, 1987.

[144] P. V. Holmes, D. Caroline Blanchard, R. J. Blanchard, L. S. Brady, and J. N. Crawley, "Chronic social stress increases levels of preprogalanin mRNA in the rat locus coeruleus," Pharmacology Biochemistry and Behavior, vol. 50, no. 4, pp. 655-660, 1995.

[145] J. C. Morales-Medina, Y. Dumont, and R. Quirion, "A possible role of neuropeptide Y in depression and stress," Brain Research, vol. 1314, pp. 194-205, 2010.

[146] G. Griebel and F. Holsboer, "Neuropeptide receptor ligands as drugs for psychiatric diseases: the end of the beginning?" Nature Review Drug Discovery, vol. 11, pp. 462-478, 2012. 

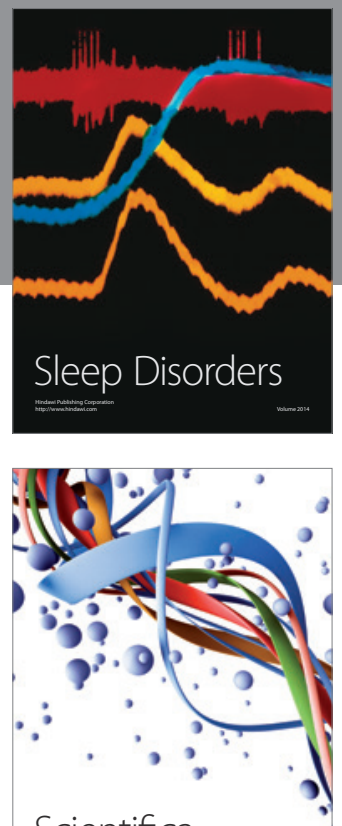

Scientifica
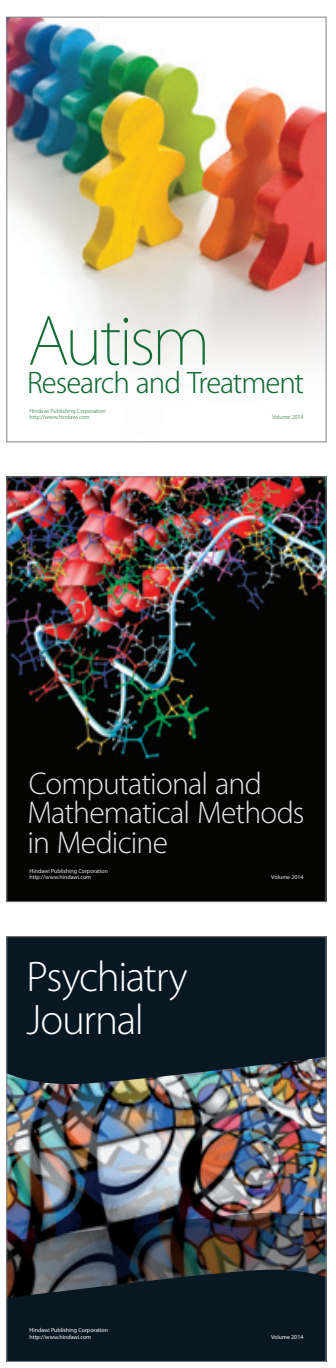
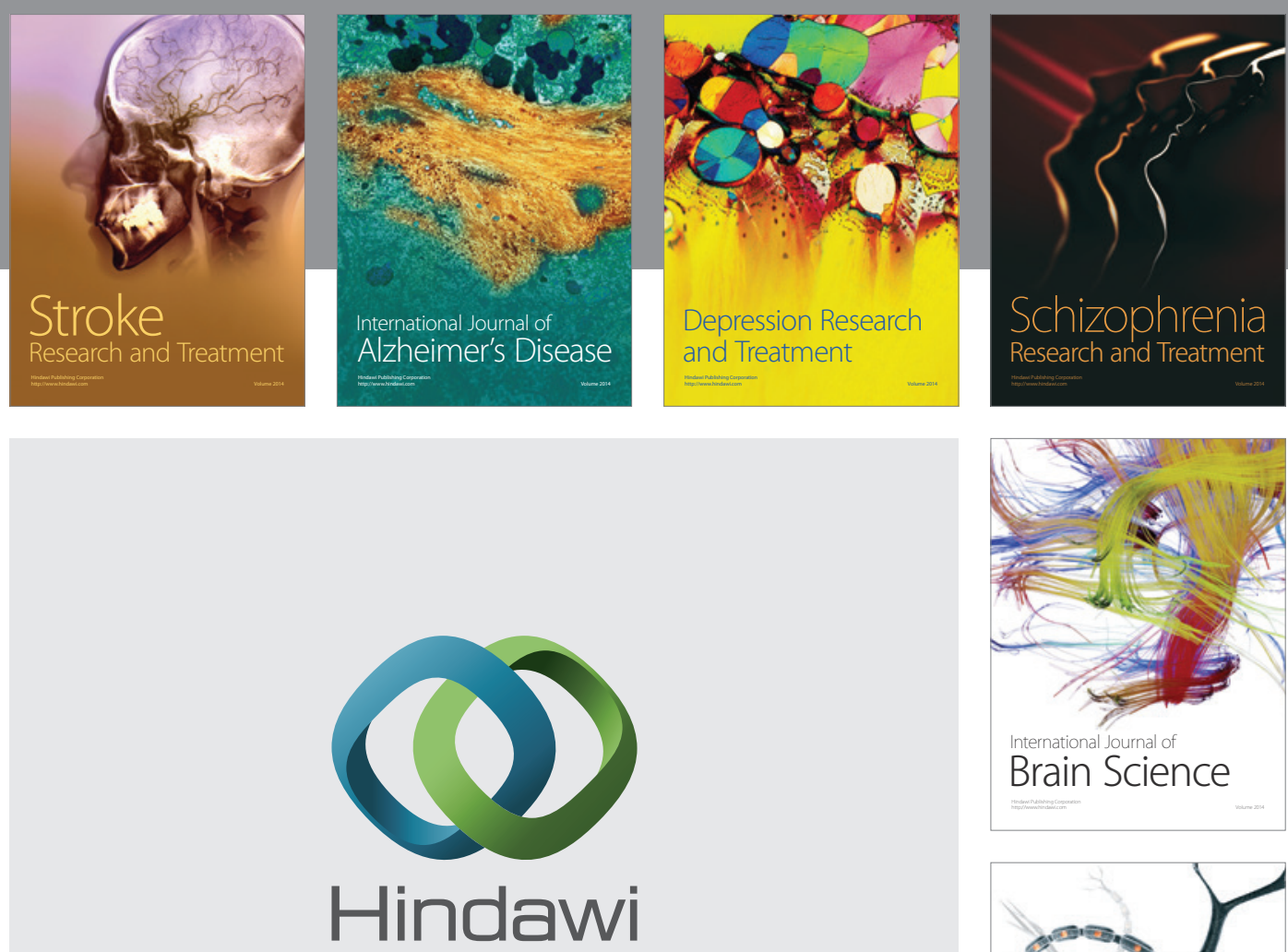

Submit your manuscripts at

http://www.hindawi.com
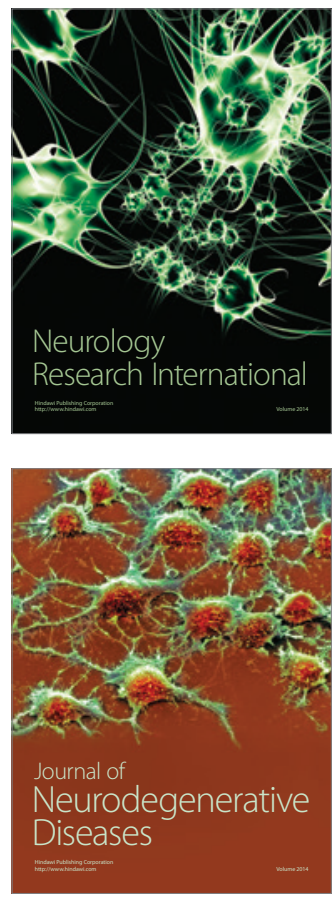

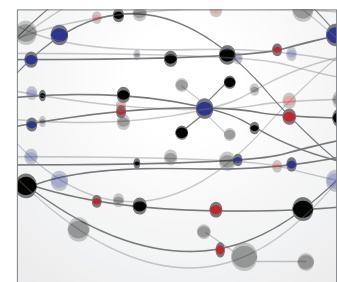

The Scientific World Journal
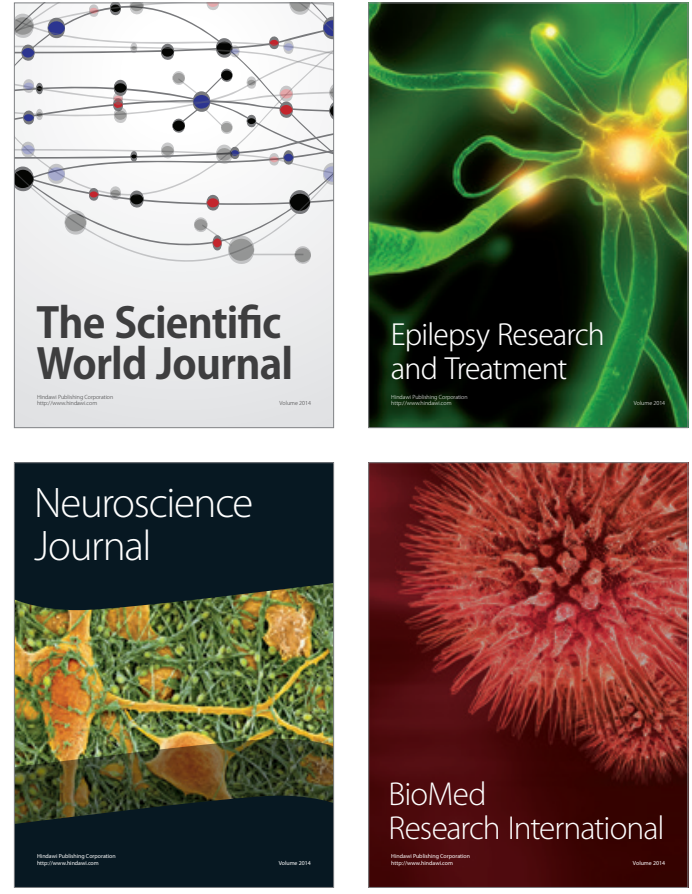

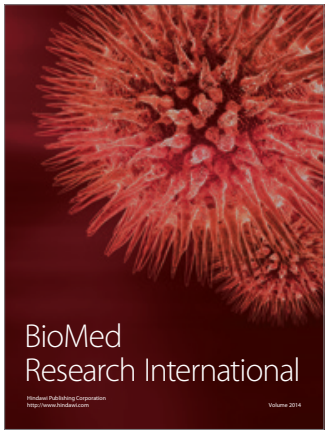

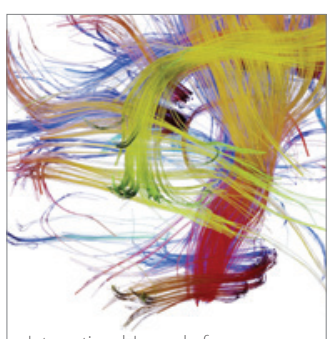

Brain Science

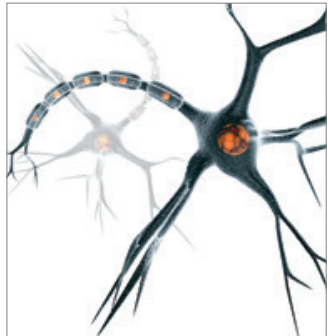

Neural Plasticity
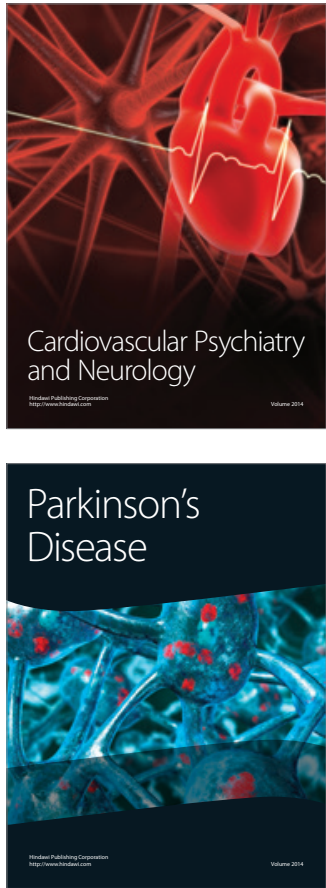